\title{
Nucleophile Substitution von Nitrit in Nitrobenzolen, Nitrobiphenylen und Nitronaphthalinen
}

\author{
Franz Effenberger*, Markus Koch ${ }^{1)}$ und Willi Streicher ${ }^{2)}$ \\ Institut für Organische Chemie der Universität Stuttgart, \\ Pfaffenwaldring 55, D-7000 Stuttgart 80 \\ Eingegangen am 16. Mai 1990
}

Key Words: Nitro compounds, aromatic / Nitrite exchange by nucleophiles

\begin{abstract}
Nucleophilic Substitution of Nitrite in Nitrobenzenes, Nitrobiphenyls, and Nitronaphthalenes
Aromatic compounds, accessible only by multistep procedures, can be synthesized easily by nucleophilic substitution of nitrite in nitrobenzenes, nitrobiphenyls, and nitronaphthalenes. Thus, meta-substituted phenols 3,4 , and 7 are obtained from 1,3-dinitrobenzene (1) and meta-substituted nitrobenzenes 6, as well as 3,5-disubstituted phenols 10 and 5-substituted resorcinol derivatives $\mathbf{1 1}$ from 3,5-disubstituted nitro-

$13,15,17,19,21,23,24$, and 26 are also available by nitrite exchange from the corresponding easily accessible dinitrobiphenyls $16,18,20,22$, and 25 . A nitrite exchange with nucleophiles is easily possible in the 1,5-disubstituted naphthalenes 29, 34, while in the case of the 1,8-disubstituted naphthalenes 31, 36 only the chloro derivative $\mathbf{3 6}$ undergoes this exchange under much stronger conditions in low yield.
\end{abstract} benzenes 9 . The unsymmetrically substituted nitrobiphenyls
In der voranstehenden Arbeit ${ }^{3)}$ haben wir über die nucleophile Substitution aromatischer Nitro-Gruppen durch Fluorid und über die Darstellung einiger auf diese Weise zugänglicher Fluoraromaten berichtet. Die einfache Synthesemöglichkeit vieler Nitro- und Polynitro-substituierter Aromaten durch direkte elektrophile Aromatennitrierung veranlaßte uns, das Synthesepotential unterschiedlicher Typen von Nitroaromaten im Hinblick auf den nucleophilen Austausch von Nitro-Gruppen zu untersuchen.

Die nucleophile Substitution von Nitro-Gruppen in Aromaten, die durch ortho- oder para-ständige Akzeptor-Substituenten $\left(\mathrm{NO}_{2}\right.$, $\mathrm{CN}, \mathrm{COR}, \mathrm{u} . \mathrm{a}$.) gegenüber einem nucleophilen Austausch aktiviert sind, ist schon lange bekannt, dagegen ist dic nucleophile Substitution von weniger aktivierten Nitro-Gruppen erst in neucrer Zeit (in dipolar aprotischen Lösungsmitteln) gelungen ${ }^{4}$. Als Nucleophile wurden dabei vor allem Sauerstoff- sowie Schwefel-Nucleophile ${ }^{5 \text {, }}$ Stickstoff- ${ }^{6}$, Kohlenstoff- ${ }^{77}$ und Phosphor-Nucleophile ${ }^{8)}$, Halogenide $^{3.9\}}$, Hydrid ${ }^{109}$ und Nitrit ${ }^{17)}$ eingesetzt. Eine intramolekulare Substitution aromatischer Nitro-Gruppen läuft erwartungsgemäB dann bevorzugt $a b$, wenn die Möglichkeit zur Bildung energetisch begünstigter 6- oder 5-Ringe besteht ${ }^{12}$. Die Darstellung von Polyarylethern durch Umsetzung von Aromaten, die aktivierte NitroGruppen besitzen, mit Diphenolaten ${ }^{13)}$ ist eine interessante präparative Anwendung dieser nucleophilen Substitution.

\section{Umsetzungen meta-ständig substituierter Nitrobenzole mit Alkoholaten}

Die Darstellung meta-substituierter Phenole gelingt weder über eine direkte elektrophile Substitution von Phenolen, da hierbei die Zweitsubstitution in ortho- oder para-Position erfolgt, noch über eine direkte Hydroxylierung von Aromaten mit Substituenten zweiter Ordnung, da alle bekannten Hydroxylierungs-Agenzien dafür ein zu geringes elektrophiles Potential besitzen ${ }^{14)}$; meta-substituierte Phenole können daher nur über Umwege erhalten werden. Ein besonders interessantes Beispiel, das diese Problematik aufzeigt, ist die Gewinnung des 3-Nitrophenols. Da die Alkali-Schmelze bei der 3-Nitrobenzolsulfonsäure nicht zu dem gewünschten Phenol führt ${ }^{15)}$, wird diese einfache Verbindung meist über das DiazoniumSalz hergestellt. In Patenten wird die Darstellung von 3-Nitrophenol ausgehend von 1-Chlor-3-nitrobenzol mit Wasser bei $420^{\circ} \mathrm{C}$ über aktivierter Kieselsäure ${ }^{16 a)}$ oder ausgehend von 3,3'-Dinitrodiphenylsulfon mit Natronlauge bei $300-320^{\circ} \mathrm{C}^{16 \text { b) }}$ beschrieben. In neuerer Zeit wurden durch nucleophile Photosubstitutionen aus 3Nitroanisol mit Butylamin ${ }^{17 a)}$ oder aus 3-Fluor-4-methoxynitrobenzol $^{17 b)}$ in alkalischer Lösung die entsprechenden 3-Nitrophenole erhalten.

3-Nitroanisol ist zwar durch direkte nucleophile Substitution aus meta-Dinitrobenzol (1) mit Kaliummethanolat in Hexamethylphosphorsäureamid (HMPA) ${ }^{4 c)}$ oder mit Chlorbenzol/Methyltrioctylammoniumchlorid ${ }^{14 d)}$ in guter Ausbeute zugänglich, Schwierigkeiten bereitet jedoch die Ether-Spaltung. In japanischen Patenten wird die Nitrit-Substitution in 1,3-Dinitrobenzol (1) mit Alkoholen oder Alkoholaten unter verschiedenen Bedingungen zu 1-Alkoxy3 -nitrobenzolen ${ }^{(8)}$ und deren Spaltung mit 25 proz. wäBrigen Lösungen von Halogenwasserstoff bei $140^{\circ} \mathrm{C}$ im Autoklaven zu 3Nitrophenol beschrieben ${ }^{19)}$.

Schema 1<smiles>O=[14C]([O-])Oc1cccc([N+](=O)[O-])c1</smiles>

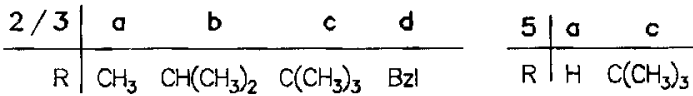

$$
\begin{aligned}
& \begin{array}{l|ll}
2 & 2^{\prime} & \mathrm{Bz}
\end{array}=\mathrm{CH}_{2} \mathrm{Ph}
\end{aligned}
$$


Da die mit Säure katalysierte Spaltung des Isopropyl- und tert-Butylethers und die Hydrogenolyse des Benzylethers leichter erfolgen sollte, haben wir die beschriebenen Umsetzungen von $1 \mathrm{mit}$ Isopropylat, tert-Butylat und Benzylalkoholat eingehend untersucht.

Bei der Umsetzung von 1 mit Alkoholaten 2 verlief die Nitrit-Substitution in Tetramethylharnstoff (TMH) zu 1Alkoxy-3-nitrobenzolen 3 erwartungsgemäß mit Natriummethanolat ${ }^{\circ}\left(\mathbf{2}^{\prime}\right)$ am besten, während mit Natrium- $\left(\mathbf{2}^{\prime} \mathbf{b}^{\prime}\right)$ oder Kaliumisopropylat (2b) unter ähnlichen Reaktionsbedingungen nur schlechte Ausbeuten an 3-Isopropoxy-3-nitrobenzol (3b) erzielt wurden (Tab. 1); offensichtlich wirkt Isopropylat in einer Konkurrenzreaktion ebenso wie Methanolat auch als Reduktionsmittel ${ }^{20)}$.

Tab. 1. Umsetzung von 1,3-Dinitrobenzol (1) mit Alkoholaten 2 in TMH unter Schutzgas $\left(\mathbf{N}_{2}, \mathrm{Ar}\right)$ zu 1-Alkoxy-3-nitrobenzolen 3, 3Nitrophenol (4) und 1,3-Dinitro-4-hydroxy- (5a) bzw. -5-tert-butoxybenzol (5e)

\begin{tabular}{|c|c|c|c|c|c|c|}
\hline 2 & $\begin{array}{l}\text { proz. } \\
\text { Mol- } \\
\text { verh. } \\
1: 2\end{array}$ & $\begin{array}{c}\text { Reakt } \\
\text { zeit } \\
h\end{array}$ & $\begin{array}{l}\text { tions- } \\
\text { temp. } \\
{ }^{\circ} \mathrm{C}\end{array}$ & $\begin{array}{l}\text { Pro } \\
\text { Aus } \\
1 \approx\end{array}$ & $\begin{array}{l}\text { dukte } \\
\text { b. } \% \\
\mathbf{3}\end{array}$ & Bemerkungen \\
\hline $2 \mathbf{a}^{\prime}$ & $1: 1.3$ & 20 & RT & $12^{b}$ & $3 \mathbf{a}: 67^{b}$ & \\
\hline $2 b^{\prime}$ & $1: 1.3$ & 20 & RT & 416 & $3 \mathbf{b}: 28^{b}$ & \\
\hline $\begin{array}{l}2 b \\
2 b \\
2 b\end{array}$ & $\begin{array}{l}1: 1.5 \\
1: 1.4 \\
1: 1\end{array}$ & $\begin{array}{l}24 \\
24 \\
24\end{array}$ & $\begin{array}{l}\text { RT } \\
\text { RT } \\
\text { RT }\end{array}$ & $\begin{array}{r}- \\
19 e \\
4 e\end{array}$ & $\begin{array}{l}3 b: 23^{c} \\
3 b: 30^{c} \\
3 b: 32^{c}\end{array}$ & $\begin{array}{l}\text { unter Sauerstoff } \\
\text { mit } 18-K \text { rone-6 }\end{array}$ \\
\hline $\begin{array}{l}2 c^{\prime} \\
2 c^{\prime}\end{array}$ & $\begin{array}{l}1: 1.2 \\
1: 1.4\end{array}$ & $\begin{array}{r}22 \\
6\end{array}$ & $\begin{array}{r}R T \\
0\end{array}$ & $\begin{array}{r}1 b \\
23^{b}\end{array}$ & $\begin{array}{l}3 c: 0.2^{b} \\
3 c:-\end{array}$ & $\begin{array}{l}+4^{b}(11 \%) \\
+4^{b}(7 \%),+5 a^{b}(20 \%)\end{array}$ \\
\hline $\begin{array}{l}2 c \\
2 c \\
2 c \\
2 c \\
2 c \\
2 c \\
2 c\end{array}$ & $\begin{array}{l}1: 1 \\
1: 1 \\
1: 1 \\
1: 0.8 \\
1: 2 \\
1: 1.4 \\
1: 1.1\end{array}$ & $\begin{array}{l}24 \\
24 \\
24 \\
48 \\
18 \\
24 \\
24\end{array}$ & $\begin{array}{r}0 \\
50 \\
100 \\
0 \\
0 \\
0 \\
R T\end{array}$ & $\begin{array}{l}22^{c} \\
13^{\circ} \\
16^{\circ} \\
28^{\circ} \\
11^{\circ} \\
21^{\circ} \\
66^{\circ}\end{array}$ & $\begin{array}{l}\text { 3c: Spur } \\
3 c: \text { Spur } \\
\text { 3c: Spur } \\
3 c:- \\
3 c:- \\
3 c:- \\
3 c:-\end{array}$ & $\begin{array}{l}+5 a^{c}(3 \%) \\
+5 a^{c}(2 x) \\
+5 a^{d}(5 \%),+5 c^{d}(19 \%) \\
\text { mit } 18-K r o n e-6 \\
\text { mit 18-Krone-6 } \\
\text { unter Sauerstoff }\end{array}$ \\
\hline $\begin{array}{l}2 d \\
2 d \\
2 d\end{array}$ & $\begin{array}{l}1: 1 \\
1: 2 \\
1: 1.7\end{array}$ & $\begin{array}{r}24 \\
8 \\
6\end{array}$ & $\begin{array}{l}\text { RT } \\
\text { RT } \\
\text { RT }\end{array}$ & $\begin{array}{l}46^{c} \\
13^{\circ} \\
38^{\circ}\end{array}$ & $\begin{array}{l}3 d: 24^{c} \\
3 d: 47^{b} \\
3 d: 49^{c}\end{array}$ & unter sauerstoff \\
\hline
\end{tabular}

a) Nicht umgesetztes Edukt. - b) Nach chromatographischer Aufarbeitung. - ${ }^{\text {c) }}$ Gaschromatographisch bestimmt. - d) Nach chromatographischer Aufarbeitung und Reinigung der 1. Fraktion mittels MPLC. - ${ }^{\text {el }}$ Reaktion war nach $4 \mathrm{~h}$ beendet. - RT: Raumtemperatur.

Obwohl diese Konkurrenzreaktion bei der Umsetzung von 1 mit tert-Butylat, das kein als Hydrid abspaltbares $\alpha$ H-Atom enthält, nicht mehr erfolgen kann, resultierten bei unseren Umsetzungen von 1 mit Natrium- $\left(2 c^{\prime}\right)$ oder Kalium-tert-butylat $(2 \mathrm{c})$ bei $0-100^{\circ} \mathrm{C}$ - auch nach langen Reaktionszeiten - lediglich Spuren an Substitutionsprodukt 3c, sowie 3-Nitrophenol (4) und 2,4-Dinitrophenol (5a); mit der doppelt molaren Menge $2 \mathrm{c}$ erhielten wir nach 18 Stunden bei $0^{\circ} \mathrm{C}$ neben kleinen Mengen 5 a noch $19 \%$ 2,4Dinitro-1-tert-butoxybenzol (5c) (Tab. 1).

Die Bildung von Phenolen wurde auch bei nucleophilen Substitutionen von 3- und 4-Halogenpyridinen mit tert-Butylaten beobachtet ${ }^{21)}$, ebenso wie die nucleophile Substitu- tion von Wasserstoff-Atomen bei der Umsetzung von 3-substituierten Nitrobenzolen mit Kaliumhydroxid ${ }^{22)}$. Bei einem Angriff eines Nucleophils in 4-Position von 1 ist die Bildung eines Meisenheimer-Komplexes energetisch besonders begünstigt, die Weiterreaktion zu den Substitutionsprodukten 5 erfolgt dann jedoch nicht durch Hydrid-Austritt, sondern über eine Oxidation des Meisenheimer-Komplexes durch noch vorhandene Ausgangsverbindung 1 zum Radikal und anschließende Deprotonierung durch Alkoholat ${ }^{23}$.

Bei der Reaktion von 1 mit überschüssigem Kaliumbenzylalkoholat (2d) erhielten wir bereits nach 8 Stunden bei Raumtemperatur das Substitutionsprodukt 3d in 47 proz. Ausbeute; offensichtlich laufen aber auch hier Nebenreaktionen ab, die eine höhere Ausbeute an 3d verhindern.

Nitroaromaten reagieren mit Nucleophilen leicht unter Elektronentransfer zu Radikalanionen, die in der Regel jedoch nicht unter Austritt von Nitrit im Sinne einer $\mathrm{S}_{\mathrm{RN}} 1$ Reaktion $^{24)}$ weiterreagieren, sondern Reduktionsprodukte als wichtigste Folgeprodukte ergeben; diese unerwünschte Reaktion kann durch Reoxidation, z.B. mit Sauerstoff oder Peroxid-Ionen vermieden werden ${ }^{25}$.

Die von uns durchgeführten Umsetzungen von 1 mit verschiedenen Alkoholaten 2 - einmal unter Schutzgas und zum anderen unter Durchleiten eines Sauerstoff-Stromes liefen vergleichbar rasch $a b$, jedoch waren in Gegenwart von Sauerstoff die Nebenreaktionen (Reduktionen) deutlich weniger ausgeprägt, so daß insgesamt höhere Umsatzausbeuten an Substitutionsprodukten erzielt wurden (Tab. 1).

Bei Versuchen zur Optimierung der Reaktion mit Benzylalkohol in Gegenwart von Kaliumhydroxid unter Sauerstoff bei $50^{\circ} \mathrm{C}$ haben sich TMH als bestes Lösungsmittel und Tetrabutylammoniumbromid (TBB) als der am besten geeignete Phasentransfer-Katalysator erwiesen.

Wir haben nun verschiedene meta-substituierte Nitrobenzole 6 mit Benzylalkohol/Kaliumhydroxid in TMH unter Sauerstoff umgesetzt mit dem Ziel, einerseits die erforderliche Aktivierung durch meta-Substituenten für den nucleophilen Austausch von Nitro-Gruppen zu ermitteln und andererseits bei Nitroaromaten, die weitere nucleofuge Austrittsgruppen besitzen, zu untersuchen, welche Gruppen substituiert werden (Tab. 2).

Schema 2

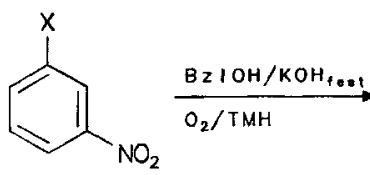

6

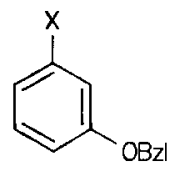

7

$$
\begin{array}{c|cccccc}
6 / 7 & \mathrm{a} & b & c & d & e & f \\
\hline X & \mathrm{~F} & \mathrm{C} & \mathrm{Br} & \mathrm{FhSO} & \mathrm{CN}
\end{array}
$$

Reaktionen von 3-Fluornitrobenzol (6a) mit Nucleophilen sind schon lange bekannt, mit Sauerstoff-Nucleophilen wurde ausschließlich Substitution des Fluor-Atoms gefunden ${ }^{26}$. Unter den in Tab. 2 aufgeführten Bedingungen er- 
folgte neben der Substitution von Fluorid unter Bildung von 3-(Benzyloxy)nitrobenzol (3d) in geringem Maße auch Substitution von Nitrit zu 3-(Benzyloxy)fluorbenzol (7a).

Tab. 2. Umsetzung von 1-substituierten 3-Nitrobenzolen 6 mit Benzylalkohol/Kaliumhydroxid (2d) in TMH unter Sauerstoff zu 1substituierten 3-(Benzyloxy)fluorbenzolen 7 und 3-(Benzyloxy)nitrobenzol $(\mathbf{3 d})^{\mathbf{a})}$

\begin{tabular}{|c|c|c|c|c|c|c|c|c|c|}
\hline \multirow{2}{*}{$\frac{6}{6 a}$} & \multicolumn{2}{|c|}{$\begin{array}{l}\text { Reaktions- } \\
\text { zeit temp. } \\
\text { h } \mathrm{c}\end{array}$} & \multicolumn{2}{|c|}{$\begin{array}{c}\text { Produkte.. } \\
\text { Ausb. } \\
(\mathbf{x})\end{array}$} & \multicolumn{2}{|c|}{$\begin{array}{l}\text { Reaktion } \\
\text { zeit } \\
6 \quad h\end{array}$} & \multirow{2}{*}{$\begin{array}{l}\text { s- } \\
\text { temp. } \\
{ }^{\circ} \mathrm{C}\end{array}$} & \multicolumn{2}{|c|}{$\begin{array}{c}\text { Produkte } \\
\text { Ausb. } \\
(x)\end{array}$} \\
\hline & $\begin{array}{l}5 \\
3\end{array}$ & $\begin{array}{l}\text { RT } \\
50\end{array}$ & $\begin{array}{r}3 d \\
+7 a\end{array}$ & $\begin{array}{r}87 \\
7\end{array}$ & $6 d$ & 40 & & $7 d^{b}$ & \\
\hline $6 b$ & 24 & 50 & $7 b$ & 97 & $6 e$ & 8 & $R T$ & $7 e$ & 95 \\
\hline $6 c$ & 24 & 50 & $7 c$ & 94 & $6 f$ & 20 & $R T$ & $7 f$ & 15 \\
\hline
\end{tabular}

a) Aufarbeitung und physikalische Daten siehe Exp. Teil. - ${ }^{\text {b) Keine }}$ voliständige Umsetzung von 6d (nach GC); es wurden 7d sowie als weitere Produkte 3,3'-Diiodazoxybenzol und Nitrobenzol mittels GC/MS bestimmt. - RT: Raumtemperatur.

Umsetzung von 3-Chlornitrobenzol (6b) mit Nucleophilen erfolgen in der Regel unter Substitution des ChlorAtoms ${ }^{27}$, lediglich sterisch gehinderte Verbindungen, wie z.B. 2-Chlor-6-nitrotoluol reagieren auch unter Substitution der Nitro-Gruppe - z.B. mit Mercaptoessigester und Lithiumhydroxid in HMPA ${ }^{28}$. Wir erhielten auch bei den sterisch nicht gehinderten 3-Chlor- (6b) und 3-Bromnitrobenzolen (6c) mit Benzylalkohol/Kaliumhydroxid nahezu ausschließlich Nitro-Substitution zu 3-(Benzyloxy)chlor- (7b) bzw. -brombenzol (7c). In flüssigem Ammoniak wird bei der Umsetzung von meta-Halogennitrobenzolen $(\mathrm{Hal}=\mathrm{Cl}, \mathrm{Br}$, I) mit Kaliumhydroxid bei $-33^{\circ} \mathrm{C}$ auch eine Hydroxylierung zu Halogennitrophenolen beschrieben, wobei die OHGruppe in ortho- oder para-Stellung zur Nitro-Gruppe sitzt $^{22)}$.

Unsere Umsetzungen von 3-Iodnitrobenzol (6d) mit Benzylalkohol/Kaliumhydroxid führten auch nach 40 Stunden bei $50^{\circ} \mathrm{C}$ zu keinem vollständigen Umsatz von $6 \mathrm{~d}$; GC/MSAnalysen zeigten, daß nahezu ausschließlich Nitro-Substitution zu 3-(Benzyloxy)iodbenzol (7 d) erfolgte, daneben aber entstanden - trotz Arbeiten unter Sauerstoff - auch geringe Mengen an 3,3'-Diiodazoxybenzol und Nitrobenzol, die als Folgeprodukte des 3-Iodnitrobenzol-Radikalanions anzusehen sind; die Abspaltung von Iodid aus diesem Radikalanion ist bekannt ${ }^{29}$.

Wiederum eindeutig verlief die Umsetzung von 3-Nitro(phenylsulfonyl)benzol (6e) mit Benzylalkohol/Kaliumhydroxid. Bereits bei Raumtemperatur erfolgte Substitution der Nitro-Gruppe zu 3-(Benzyloxy)(phenylsulfonyl)benzol (7e), obwohl auch Benzolsulfinat eine gute Austrittsgruppe ist.

Reaktionen von 3-Nitrobenzonitril (6f) mit Nucleophilen (2,2,2-Trifluorethanolat), die ebenfalls unter Substitution der Nitro-Gruppe verlaufen, sind bekannt ${ }^{30}$; wir erhielten aus 6f mit Benzylalkohol/Kaliumhydroxid bereits bei Raumtemperatur 3-(Benzyloxy)benzonitril (7f), allerdings mit nur 15\% Ausbeute.
Die Umsetzungen von 3-Nitrobenzoesäure, deren Methylester und Amiden sowie von 3-Nitrobenzo- und -acetophenon und 3-Nitrobenzaldehyd führten zu keinen eindeutigen Substitutionsprodukten, da unter den angewandten Bedingungen in stark basischem Medium offensichtlich andere Reaktionen dieser Carbonylverbindungen bevorzugt ablaufen.

Die in Schema 2 und Tab. 2 aufgeführten Reaktionen stellen insgesamt einen einfachen Zugang zu meta-substituierten Phenolen dar, die bislang häufig nur über Vielstufenreaktionen zugänglich sind.

\section{Umsetzungen 1,3,5-substituierter Nitrobenzole mit Benzylalkohol/Kaliumhydroxid}

Die Substitution von Nitro-Gruppen in 1,3,5-trisubstituierten Benzolen mit Methanolat ${ }^{46,31,32)}$ oder Thiophenolat bzw. Piperidin/Methanol ${ }^{33}$ ist bekannt. Wir haben jetzt die mit ein bis drei Nitro-Substituenten 1,3,5-trisubstituierten Benzole 9 mit Benzylalkohol/Kaliumhydroxid in TMH unter Sauerstoff umgesetzt mit dem Ziel, auf diesem Wege sonst nur schwer zugängliche 5-substituierte Resorcine darzustellen (Tab. 3).

Schema 3

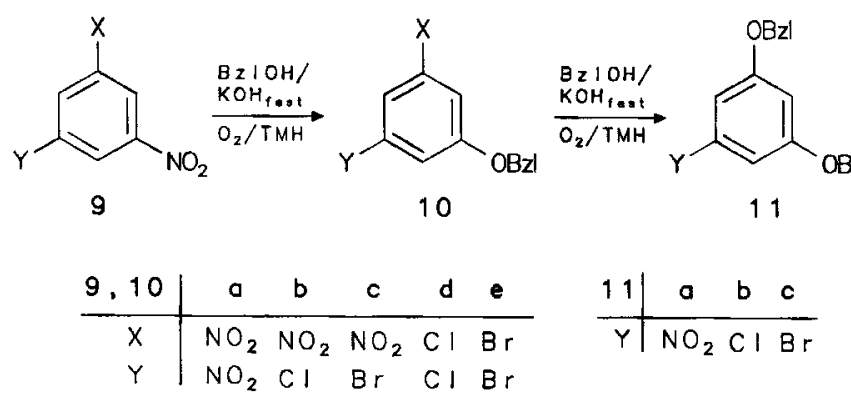

Tab. 3. Umsetzung von 1,3,5-trisubstituierten Benzolen 9 und 10 mit Benzylalkohol/Kaliumhydroxid in TMH unter Sauerstoff

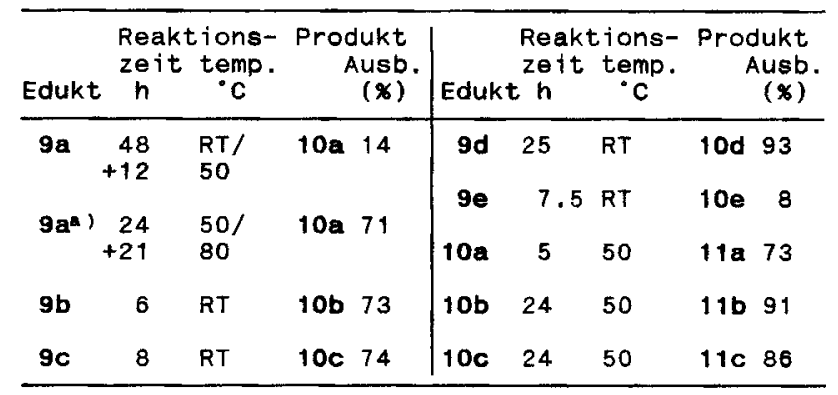

a) Mit Kaliumcarbonat anstelle von Kaliumhydroxid. - RT: Raumtemperatur.

Obwohl bei 1,3,5-Trinitrobenzol (9a) wegen der erhöhten Aktivierung der meta-Nitro-Gruppe durch zwei NitroGruppen eine leichte Substitution durch Methanolat erfolgt ${ }^{34)}$, erhielten wir bei der Umsetzung von 9a mit Benzylalkohol/Kaliumhydroxid auch nach mehrstündigem Erhitzen auf $50^{\circ} \mathrm{C}$ keinen vollständigen Umsatz und nur $14 \%$ 
des erwarteten Substitutionsprodukts 10a. Dieser unerwartete Befund könnte mit der Bildung eines in TMH stabilen Meisenheimer-Komplexes durch Angriff eines im Überschuß vorhandenen Hydroxid-Ions in 2-Position von $9 \mathrm{a}^{35)}$ erklärt werden. Ein Ersatz von Kaliumhydroxid durch die schwächere Base Kaliumcarbonat, die sich bei der Umsetzung von 9a mit wäßrigem Methanol bewährt hat ${ }^{36)}$, führte bei der Umsetzung von 9a mit Benzylalkohol nach mehrstündigem Erhitzen auf $50-80^{\circ} \mathrm{C}$ in guter Ausbeute zu 1(Benzyloxy)-3,5-dinitrobenzol (10a), das mit Benzylalkohol/ Kaliumhydroxid in TMH unter Sauerstoff nach Erhitzen auf $50^{\circ} \mathrm{C}$ erwartungsgemäß (siehe oben) unter Substitution einer weiteren Nitro-Gruppe das 1,3-Bis(benzyloxy)-5-nitrobenzol (11 a) in guter Ausbeute ergab.

Bei 1-Chlor- (9b) und 1-Brom-3,5-dinitrobenzol (9c) erhielten wir mit Benzylalkohol/Kaliumhydroxid selektive Substitution einer Nitro-Gruppe bereits bei Raumtemperatur, die gebildeten 1-(Benzyloxy)-3-halogen-5-nitrobenzole $10 \mathrm{~b}$ und $10 \mathrm{c}$ ließen sich mit Benzylalkohol/Kaliumhydroxid bei $50^{\circ} \mathrm{C}$ wiederum zu den 1,3-Bis(benzyloxy)-5halogenbenzolen $11 \mathrm{~b}$ und $11 \mathrm{c}$ umsetzen. In keinem Fall wurde die Substitution eines Halogen-Atoms beobachtet. Damit ist in der Tat ein bequemer Zugang zu 5-Halogensubstituierten Resorcin-Derivaten 11 möglich.

Auch bei 1,3-Dichlor- (9d) und 1,3-Dibrom-5-nitrobenzol (9e) erfolgte mit Benzylalkohol/Kaliumhydroxid bei Raumtemperatur selektive Substitution der Nitro-Gruppe unter Bildung der 1-(Benzyloxy)-3,5-dihalogenbenzole 10d,e; diese Verbindungsklasse kann auf andere Weise ebenfalls nur aufwendig dargestellt werden.

\section{Umsetzungen von Dinitrobiphenylen mit Nucleophilen}

Unsymmetrisch substituierte Biphenyle erhält man in zum Teil guten Ausbeuten unter Verwendung von Phasentransfer-Katalysatoren bei der Gomberg-Bachmann-Reaktion $^{37)}$ durch Umsetzung von Diarylquecksilber-Verbindungen mit aromatischen Iod- oder Brom-Verbindungen in Gegenwart eines Palladium-Katalysators ${ }^{38)}$ oder ausgehend von Dinitrobiphenylen ${ }^{39)}$ über die Diazonium-Verbindungen und deren Reaktion mit Nucleophilen ${ }^{40}$. Demgegenüber hätte die direkte Substitution einer Nitro-Gruppe in Nitrosubstituierten Biphenylen entscheidende Vorteile. Daß dies unter besonders günstigen sterischen Voraussetzungen möglich ist, wurde bei der intramolekularen Reaktion von 2-Nitro-2'-carboxybiphenyl gezeigt ${ }^{4 b}$.

Wir haben deshalb die Möglichkeiten der nucleophilen Substitution von Nitro-Gruppen in Dinitrobiphenylen generell untersucht. Bei der Umsetzung von 4,4'-Dinitrobiphenyl (12) mit $2 a^{\prime}$ in verschiedenen dipolar-aprotischen Lösungsmitteln erhielten wir zumeist die Substitution einer Nitro-Gruppe unter Bildung von 4'-Methoxy-4-nitrobiphenyl (13a), jedoch waren Reaktionsgeschwindigkeiten und Ausbeuten an 13a in starkem Maße vom Lösungsmittel abhängig. In dem für nucleophile Substitutionen sonst mit großem Erfolg eingesetzten Dimethylformamid war auch nach 72 Stunden noch keinerlei Reaktion festzustellen, während in TMH nach einer Stunde praktisch quantitative Umsetzung zu 13a erfolgte (Tab. 4). Wir haben daher alle weiteren Umsetzungen in TMH durchgeführt.

Schema 4

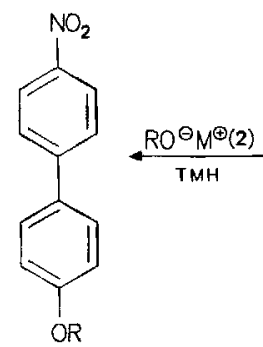

13

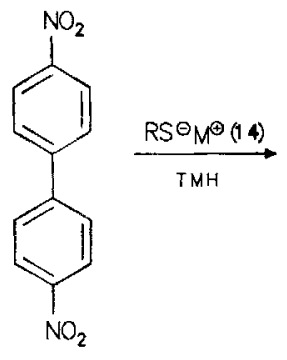

12<smiles>O=[N+]([O-])c1ccc(-c2ccc([Se])cc2)cc1</smiles>

15

\begin{tabular}{|c|c|c|c|c|c|c|c|c|}
\hline$\frac{13}{8}$ & a & $d$ & e & & $2 a^{\prime}$ & & & $2 \mathrm{e}$ \\
\hline R & $\mathrm{ICH}_{3}$ & Bzl & $\mathrm{Ph}$ & & $14 a^{\prime}$ & $2 d^{\prime}$ & $14 f^{\prime}$ & $14 \mathrm{e}$ \\
\hline 15 & a & e & $f$ & $\begin{array}{l}M \\
R\end{array}$ & $\begin{array}{l}\mathrm{No} \\
\mathrm{CH}_{3}\end{array}$ & $\begin{array}{l}\mathrm{Na} \\
\mathrm{Bzl}\end{array}$ & $\begin{array}{l}\mathrm{No} \\
\mathrm{C}_{2} \mathrm{H}_{5}\end{array}$ & $\begin{array}{c}K \\
P h\end{array}$ \\
\hline & & & $\mathrm{C}_{2} \mathrm{H}_{5}$ & & & & & \\
\hline
\end{tabular}

Tab. 4. Umsetzung von 4,4'-Dinitrobiphenyl (12) mit Alkoholaten 2, Mercaptiden und Thiolaten 14 in TMH unter Stickstoff

\begin{tabular}{|c|c|c|c|c|c|c|c|c|c|}
\hline 2 & $\begin{array}{c}\text { Reakt } \\
\text { zeit } \\
\text { h }\end{array}$ & $\begin{array}{l}\text { tons- } \\
\text { temp. } \\
{ }^{\circ} \mathrm{C}\end{array}$ & Prod & $\begin{array}{l}\text { lukt } \\
\text { Ausb. } \\
\left.(\%)^{a}\right)\end{array}$ & 14 & $\begin{array}{l}\text { Reakt } \\
\text { zeit } \\
h\end{array}$ & $\begin{array}{l}\text { tions- } \\
\text { temp. } \\
{ }^{\circ} \mathrm{C}\end{array}$ & Pro & $\begin{array}{l}\text { lukt } \\
\text { Ausb. } \\
(\%) \text { i) }\end{array}$ \\
\hline $\begin{array}{l}2 a^{\prime} \\
2 d^{\prime} \\
2 e\end{array}$ & $\begin{array}{r}1 \\
2 \\
72\end{array}$ & $\begin{array}{l}\text { RT } \\
\text { RT } \\
70\end{array}$ & $\begin{array}{l}13 \mathbf{a} \\
13 \mathbf{d} \\
13 \mathbf{e}\end{array}$ & $\begin{array}{l}98 \\
95 \\
93\end{array}$ & $\begin{array}{l}14 a^{\prime} \\
14 f^{\prime} \\
14 e\end{array}$ & $\begin{array}{r}6 \\
6 \\
72\end{array}$ & $\begin{array}{l}-2 \\
-2 \\
40\end{array}$ & $\begin{array}{l}15 a \\
15 f \\
15 e\end{array}$ & $\begin{array}{l}87 \\
72 \\
70\end{array}$ \\
\hline
\end{tabular}

a) Präparative Ausbeuten.

Die Reaktionen mit Natriummethanolat (2a') und -benzylalkoholat (2 $\left.\mathbf{d}^{\prime}\right)$ verliefen vergleichbar gut; bei Raumtemperatur wurde bereits nach 1-2 Stunden nahezu quantitativer Umsatz zu den 4'-Alkoxy-4-nitrobiphenylen 13a und 13d erzielt, während mit dem weniger reaktiven Kaliumphenolat (2e) erst nach 72 Stunden bei $70^{\circ} \mathrm{C}$ vergleichbar gute Ausbeuten an 4-Nitro-4'-phenoxybiphenyl (13e) erhalten werden. Erwartungsgemäß erfolgte die nucleophile Substitution einer Nitro-Gruppe in 12a auch mit Natriummethan- (14a') und -ethanthiolat (14f') sowie mit Kaliumthiophenolat (14e). Hierbei waren die Reaktionen mit den Natriumalkanthiolaten $14 a^{\prime}, f^{\prime}$ bei Raumtemperatur so exotherm, daß gute Ausbeuten an 4-(Alkylthio)-4'-nitrobiphenylen 15a,f nur bei Temperaturen unter $0^{\circ} \mathrm{C}$ erreicht wurden, während für eine Umsetzung mit 14e zu 4-Nitro-4'(phenylthio)biphenyl (15e) auf $40^{\circ} \mathrm{C}$ erwärmt werden mußte.

Auch bei den Reaktionen aller möglichen stellungsisomeren Nitro-nitro'-biphenyle mit $\mathbf{2} \mathbf{a}^{\prime}$ in TMH haben wir eine selektive Substitution einer Nitro-Gruppe beobachtet. Die in präparativem Maßstab durchgeführten Umsetzungen zeigen, daß die Substitution der Nitro-Gruppe in 2-Stellung eindeutig gegenüber der in 3 - und 4-Stellung $(16 \rightarrow 17$ und $\mathbf{2 0} \rightarrow \mathbf{2 1}$ ) und die Substitution der Nitro-Gruppe in 4-Stellung gegenüber der in 3-Stellung begünstigt ist $(22 \rightarrow$ be- 
vorzugt 23 als Hauptprodukt und 24 als Nebenprodukt) (Tab. 5).

Schema 5<smiles>O=[N+]([O-])c1ccc(-c2ccccc2[N+](=O)[O-])cc1</smiles>
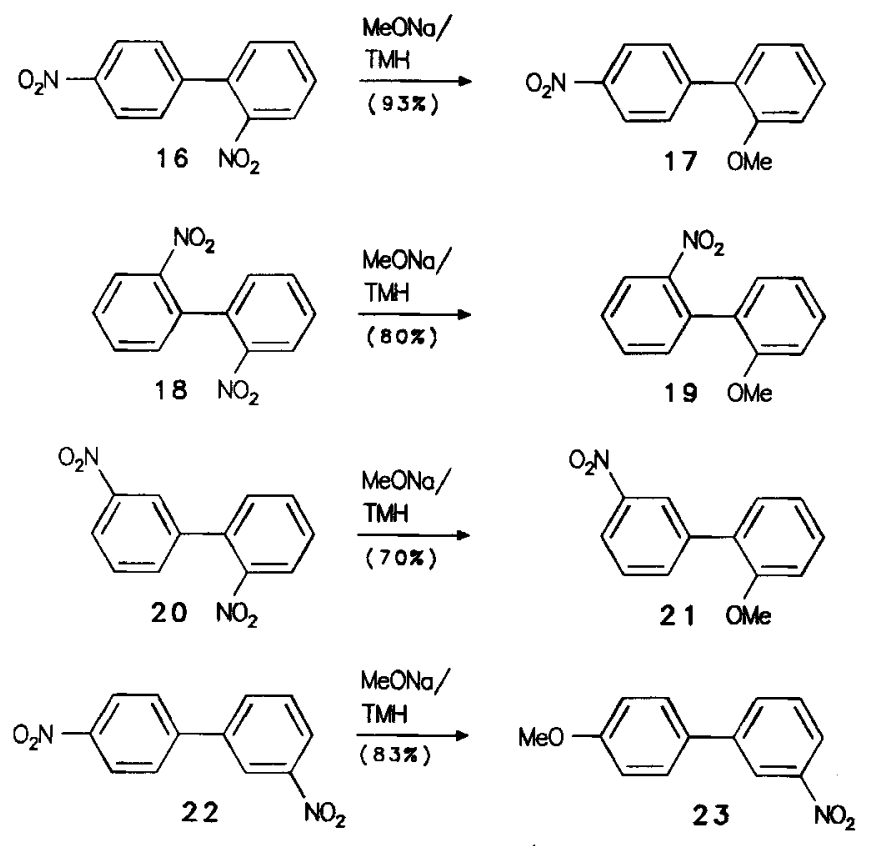

$+$
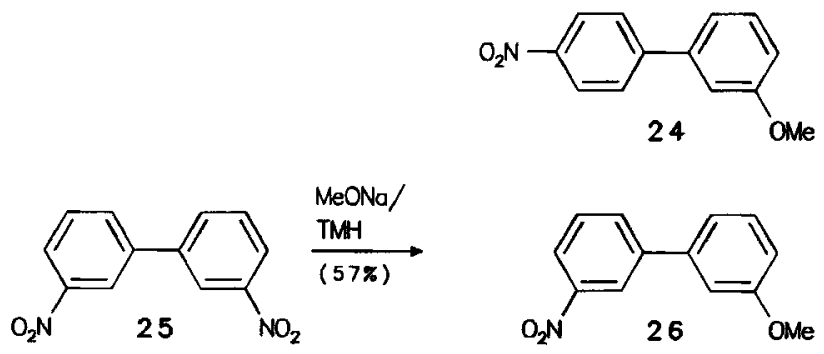

Tab. 5. Umsetzung der stellungsisomeren Dinitrobiphenyle 16, 18, 20, 22, 25 mit Natriummethanolat $\left(\mathbf{2 a}^{\prime}\right)$ in TMH bei Raumtemperatur unter Stickstoff

\begin{tabular}{|c|c|c|c|c|}
\hline $\begin{array}{l}\text { Edukt } \\
\text {-Dinitro- } \\
\text { biphenyl }\end{array}$ & $\begin{array}{l}\text { Reakt. } \\
\text { Zeit } \\
\text { h }\end{array}$ & - Pro & $\begin{array}{l}\text { dukt } \\
\text {-bipheny } 1\end{array}$ & $\begin{array}{l}\text { Ausb.a) } \\
(x)\end{array}$ \\
\hline $\begin{array}{l}2,4,-(16) \\
2,2,-(18) \\
2,3,-(20) \\
3,4=(22) \\
3,3,-(25)\end{array}$ & $\begin{array}{r}3.5 \\
21 \\
4 \\
18\end{array}$ & $\begin{array}{r}17 \\
19 \\
21 \\
23 \\
+24 \\
26\end{array}$ & $\begin{array}{l}\text { 2'-Methoxy-4-nitro- } \\
\text { 2'-Methoxy-2-nitro- } \\
\text { 2'-Methoxy-3-nitro- } \\
\text { 4, - Methoxy-3-nitro- } \\
\text { 3'-Methoxy-4-nitro- } \\
\text { 3'-Methoxy-3-nitro- }\end{array}$ & $\begin{array}{l}93 \\
80 \\
70 \\
83^{\circ)} \\
57\end{array}$ \\
\hline
\end{tabular}

${ }^{\text {at }}$ Präparative Ausbeute. $-{ }^{\text {b) }}$ Gemisch 23:24 = 7:1 (nach KapillarGC).

Nach den in Schema 4, 5 und Tab. 4, 5 aufgeführten Ergebnissen liegt mit dem nucleophilen Austausch einer NitroGruppe in den stellungsisomeren Dinitrobiphenylen eine einfache und wertvolle Methode zur Darstellung unterschiedlich substituierter Biphenyle vor.

Uber die Bestimmung der Konzentration an noch vorhandenen Edukten nach 1- bzw. 20 stündiger Umsetzung mit $2 \mathfrak{a}^{\prime}$ unter vergleichenden Bedingungen in TMH bei
Raumtemperatur haben wir folgende Reihe der Dinitrobiphenyle bezüglich ihrer Reaktivität gefunden: $12>16>$ $20>22>18>25$ (Tab. 6).

Tab. 6. Vergleichende Umsetzungen von Dinitrobiphenylen mit Natriummethanolat $\left(2 \mathbf{a}^{\prime}\right)$ in TMH bei Raumtemperatur unter Stickstoff

\begin{tabular}{|c|c|c|c|c|c|}
\hline $\begin{array}{l}\text { Edukt } \\
\text {-Dinitro- } \\
\text { bipheny } 1\end{array}$ & $\begin{array}{l}\text { noch vor } \\
\text { handenes } \\
\text { Edukt ( } \% \\
\text { nach } 2 h\end{array}$ & $\begin{array}{l}- \\
20 \mathrm{~h}\end{array}$ & $\begin{array}{l}\text { Edukt } \\
\text {-Dinitro- } \\
\text { bipheny } 1\end{array}$ & $\begin{array}{l}\text { noch vor } \\
\text { handenes } \\
\text { Edukt ( } \% \\
\text { nach } 2 h\end{array}$ & 20h \\
\hline $\begin{array}{l}4,4^{\prime}-(12) \\
2,4^{\prime}-(16) \\
2,2^{\prime}-(18)\end{array}$ & $\begin{array}{r}20 \\
40 \\
\approx 95\end{array}$ & $\overrightarrow{78}$ & $\begin{array}{l}2,3^{\prime}-(20) \\
3,4^{\prime}-(22) \\
3,3^{\prime}-(25)\end{array}$ & $\begin{array}{r}49 \\
73 \\
\approx 97\end{array}$ & $\begin{array}{r}- \\
55 \\
73\end{array}$ \\
\hline
\end{tabular}

a) Gaschromatographisch bestimmt.

Diese Reaktivitätsabstufung und Regioselektivität des Nitro-Gruppen-Austauschs in den Dinitrobiphenylen läßt sich plausibel deuten, wenn ein Additions-Abspaltungs-Mechanismus zugrundegelegt wird.

Durch die Verdrillung der beiden $\pi$-Elektronen-Systeme in Biphenylen wirken sich Substituenteneinflüsse im benachbarten Phenyl-Ring nur noch schwach aus ${ }^{41}$. So wurde z.B. bei der Umsetzung mit Piperidin gefunden, daß 4-Brom3,4'-dinitrobiphenyl ca. $12 \mathrm{mal}$ rascher als 4-Brom-3-nitrobiphenyl, jedoch $10^{5}$ mal langsamer als 2,4-Dinitrobrombenzol ${ }^{41 a)}$ reagiert.

Für die Abschätzung der Reaktivität der Nitrobiphenyle sind einmal die Mesomeriestabilisierung des MeisenheimerKomplexes durch den ungestörten Nitrophenyl-Rest zu berücksichtigen und zum anderen die Hammettschen $\sigma$ - bzw. $\sigma^{-}$-Werte für die ortho-, meta- bzw. para-NitrophenylGruppe. Bezüglich der Mesomeriestabilisierung des Meisenheimer-Komplexes muß ein Angriff des Nucleophils in ortho-l para-Position günstiger sein als in meta-Stellung $(\mathbf{1 2 , 1 6}>$ 22). Da 4,4'-Dinitrobiphenyl (12) rascher reagiert als $2^{\prime}, 4-$ Dinitrobiphenyl (16), muß man annehmen, daß die geringere sterische Hinderung in $\mathbf{1 2}$ die Bildung des MeisenheimerKomplexes begünstigt.

Aus den $\mathrm{p} K_{\mathrm{a}}$-Werten von den in 4-Stellung $\mathrm{NH}_{3}^{+}$-substituierten 4-, 3- und 2-Nitrobiphenylen ${ }^{40 b, 42)}$ haben wir unter Berücksichtigung des für die Dissoziation im jeweiligen Medium gültigen $\mathrm{Q}$-Wertes folgende $\sigma^{-}$-Werte für die Nitrophenyl-Gruppe berechnet: 4-Ammonio-4'-nitrobiphenyl: 0.29, 4-Ammonio-3'-nitrobiphenyl: 0.23 und 4-Ammonio-2'nitrobiphenyl: $0.16^{12}$. Mit diesen Werten stimmt die von uns gefundene Reaktivitätsabstufung 2'-, 4- (16) > 2'-, 3- (20) > 2'-, 2-Dinitrobiphenyl (18) überein.

Bei dem relativ geringen Substituenteneinfluß einer NitroGruppe auf die Reaktivität von Biphenylen ( $\sigma^{-}$für 2-Nitrophenyl: 0.16) stellte sich nun die Frage, ob eine NitroSubstitution auch in 4-Nitrobiphenyl (18) möglich ist. In TMH bei Raumtemperatur erfolgte mit Natriummethanolat $\left(2 a^{\prime}\right)$ eine sehr langsame Reaktion, während mit Kaliummethanolat (2a) nach 48 Stunden 4-Methoxybiphenyl (19) in 80 proz. Ausbeute isoliert werden konnte. 
Schema 6

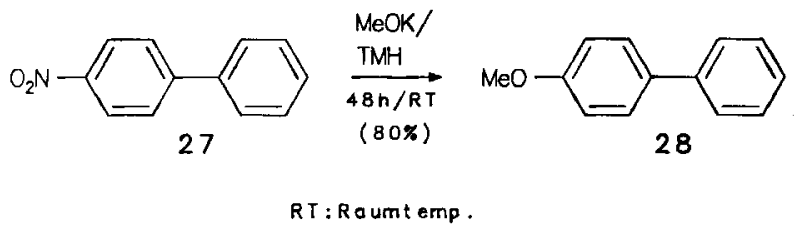

\section{Umsetzungen von Nitronaphthalinen mit Nucleophilen}

Die Möglichkeit eines nucleophilen Austauschs von Nitro-Gruppen haben wir auch an den 1,5- sowie 1,8-Dinitro(29 bzw. 31) und den 1-Chlor-5-nitro- sowie 1-Chlor-8-nitronaphthalinen (34 bzw. 36) untersucht. Das über direkte Nitrierung von Naphthalin leicht zugängliche 29 reagierte mit Benzylalkohol/Kaliumhydroxid in TMH bereits bei Raumtemperatur in sehr guten Ausbeuten zum 5-(Benzyloxy)-1-nitronaphthalin (30), bedingt durch die günstige Mesomeriestabilisierung der anionischen Zwischenstufe.

Schema 7<smiles>O=[N+]([O-])c1cccc2c([N+](=O)[O-])cccc12</smiles>

$$
\begin{aligned}
& \mathrm{BzlOH} / \\
& \mathrm{KOH}_{\text {rest }} / \\
& \underset{\mathrm{TMH}^{-}}{4 \mathrm{~h} / \mathrm{Rt}} \\
& (92 \%)
\end{aligned}
$$<smiles>O=C(OCc1ccccc1)Oc1cccc2c([N+](=O)[O-])cccc12</smiles><smiles>O=[N+]([O-])c1cccc2cccc([N+](=O)[O-])c12</smiles>

31

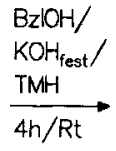<smiles>CC(=O)Oc1ccc([N+](=O)[O-])c2cccc([N+](=O)[O-])c12</smiles><smiles>O=[N+]([O-])c1cccc2ccc(O)c([N+](=O)[O-])c12</smiles>

33
R t: Roumt emp.

32 a : R $=H: 22 \%$

$32 \mathrm{~b}: \mathrm{R}=\mathrm{Bzl} ; 3 \%$

$33: 3 \%$<smiles>[R]O[C@H]1C=CC(=[N+]([O-])[O-])c2c1cccc2[N+](=O)[O-]</smiles>

A<smiles>[R]O[C@]1([2H])C=Cc2cccc([N+](=O)[O-])c2C1=[N+]([O-])[O-]</smiles>

B<smiles>[R][C@]1([N+](=O)[O-])C=C[C+]c2cccc([N+](=O)[O-])c21</smiles>

C
Über die Bildung eines Methoxy-Meisenheimer-Komplexes aus 29 bei der Umsetzung mit Methanolat und dessen langsame Weiterreaktion unter Nitrit-Austritt wurde schon berichtet ${ }^{43 a)}$.

Wesentlich komplizierter verlief die Umsetzung von $\mathbf{3 1}$ unter analogen Bedingungen; nach 4stündiger Reaktionsdauer war zwar kein Edukt mehr nachweisbar, es konnten jedoch keine Nitro-Gruppen sondern nur die WasserstoffAtom-Substitutionsprodukte $\mathbf{3 2}$ und $\mathbf{3 3}$ in zum Teil nur minimalen Mengen aus dem Reaktionsgemisch isoliert werden.
Dieses Reaktionsverhalten kann mit der energetisch bedingten, bevorzugten Bildung der Meisenheimer-Komplexe $\mathbf{A}^{43 b, c)}$ und $\mathbf{B}$ gegenüber dem für eine Substitution erforderlichen Addukt $\mathbf{C}$ gedeutet werden.

Das durch Chlorierung von 1-Nitronaphthalin leicht darstellbare 34 besitzt eine vergleichbare Aktivierung wie 4Chlornitrobenzol ${ }^{44)}$, so daß bei der Reaktion mit Alkoholat eine Chlorid-Substitution zu erwarten war. Wir erhielten jedoch bei der Umsetzung von $34 \mathrm{mit}$ Benzylalkohol/Kaliumhydroxid ausschließlich Nitrit-Substitution zu 5-(Benzyloxy)-1-chlornaphthalin (35) (Ausb. 88\%).

Die analoge Reaktion zeigte die stellungsisomere Verbindung 36 mit Benzylalkohol/Kaliumhydroxid erst nach 56 stündigem Erhitzen auf $50-80^{\circ} \mathrm{C}$, wobei nur 15\% 8(Benzyloxy)-1-chlornaphthalin (37) isoliert werden konnte.

Schema 8<smiles>O=[N+]([O-])c1cccc2c(Cl)cccc12</smiles>

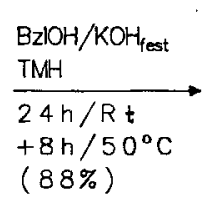<smiles>O=[N+]([O-])c1cccc2cccc(Cl)c12</smiles>

36

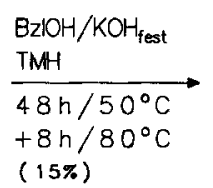

Rt: Roumtemp.<smiles>O=C(Oc1ccccc1)c1cccc(Cl)c1</smiles><smiles>O=S(=O)(O)c1cccc2cccc(Cl)c12</smiles>

37
Auch die zuletzt beschriebenen Reaktionen von Nitronapthalinen zeigen, daB insbesonders 1,5-disubstituierte Naphthaline über eine nucleophile Substitution von Nitrit auf einfache Weise zugänglich sind.

Wir danken der Deutschen Forschungsgemeinschaft und dem Fonds der Chemischen Industrie für die Unterstützung dieser Arbeit.

\section{Experimenteller Teil}

${ }^{1}$ H-NMR: Geräte T 60 und EM 360 der Firma Varian sowie WP 80 und CXP 300 der Firma Bruker, Tetramethylsilan als interner Standard. - Säulenchromatographie: Glassäulen verschiedener Größe, gepackt mit Kieselgel S, Korngröße 0.032-0.063 mm (Fa. Riedel-de Haën). - Gaschromatographie: Gerät Carlo-ErbaFractovap GI mit FID Integrator: Spectraphysics Minigrator, 0.7 bar Helium, Glaskapillare $20 \mathrm{~m} \times 2 \mathrm{~mm}$, Phasen SE 52 und 54. Temperaturprogramm: $50-250^{\circ} \mathrm{C}, 5$ und $10^{\circ} \mathrm{C} / \mathrm{min}$. Die gaschromatographisch bestimmten Ausbeuten sind auf Hydrochinondimethylether als internem Standard bezogen ${ }^{45)}$. - GC/MS: Gaschromatograph Carlo-Erba Fractovap 2151 Special (Fa. Brechbühler $A G)$, Massenspektrometer Finnigan 4023 mit IncosDatensystem 2300; Glaskapillaren: $20 \mathrm{~m}$, Phasen: SE 54 und $\mathrm{OV}$ 1701; Bedingungen CI, Methan. - Präparative Mitteldruck-Flüssigkeitschromatographie (MPLC): Nach Glatz ${ }^{46)}$ entwickeltes System; Säulen jeweils gefüllt mit Kieselgel (Fa. Merck, 0.015-0.025 $\mathrm{mm}$ ) a) $25 \times 2.4 \mathrm{~cm}, 6300$ theoretische Böden, b) $40 \times 4.5 \mathrm{~cm}$, 
6300 theoretische Böden; mobile Phase: Petrolether/Ethylacetat (PE/EE); Detektor: UV-Spektrochrom (Fa. Gilson).

TMH = Tetramethylharnstoff. - TBB $=$ Tetrabutylammoniumbromid.

I) Umsetzung von 1,3-Dinitrobenzol (1) mit Alkoholaten 2 in TMH (zu Tab. 1). - Allgemeines: In ausgeheizten Kolben wurden 1 und $\mathbf{2}$ jeweils in TMH unter Stickstoff oder Argon gelöst, i. Vak. entgast und wieder mit Schutzgas begast. $\mathrm{Zu}$ der Lösung von 1 tropfte oder spritzte man die Lösung von 2, rührte das Gemisch bei der angegebenen Temp. und goß es nach der angegebenen Zeit auf Eis. Nach Ansäuern mit konz. $\mathrm{HCl}$ wurde mit Dichlormethan oder Diethylether ausgeschüttelt, die organische Phase mit Magnesiumsulfat getrocknet, cingeengt und der Rückstand an Kieselgel mit PE/EE (4:1) chromatographiert. Bei den Umsetzungen unter Sauerstoff

Tab. 7. Umsetzung von 1,3-Dinitrobenzol (1) mit Alkoholaten 2 in TMH unter Schutzgas $\left(\mathrm{N}_{2}, \mathrm{Ar}\right)$ zu 1-Alkoxy-3-nitrobenzolen 3, 3Nitrophenol (4) und 1,3-Dinitro-4-hydroxy- (5a) bzw. -5-tert-butoxybenzol $(5 \mathrm{c})$

\begin{tabular}{|c|c|c|c|c|c|}
\hline $\begin{array}{l}1 \\
9 \\
(\mathrm{mmol})\end{array}$ & 2 & $\underset{\text { (mmo } 1)}{g}$ & $\begin{array}{l}\text { TMH } \\
m ?\end{array}$ & $\begin{array}{l}\text { Produkte } \\
\text { Ausb. } g^{\text {a }} \\
1^{01} \mathbf{3}^{-}\end{array}$ & Bemerkungen \\
\hline $\begin{array}{l}1.44 \\
(8.5)\end{array}$ & $2 a^{\prime}$ & $\begin{array}{l}0.60 \\
(11.1)\end{array}$ & 20 & $\begin{array}{rl}0.17 & \mathbf{3 a} \\
& 0.88\end{array}$ & $\begin{array}{l}\text { 3a: Schmp. } 90^{\circ} \mathrm{C} \\
\left.\text { (Lit.47) } 90^{\circ} \mathrm{C}\right) \text {. }\end{array}$ \\
\hline $\begin{array}{l}1.15 \\
(6.8)\end{array}$ & $2 b^{3}$ & $\begin{array}{l}0.70 \\
(8.5)\end{array}$ & 20 & $\begin{array}{rl}0.49 & 3 \mathrm{~b} \\
& 0.35\end{array}$ & $\begin{array}{l}\text { 3b: gelbl. } 61 \mathrm{c}) \\
(\mathrm{Lit.49}) \mathrm{Sdp} .258- \\
\left.259^{\circ} \mathrm{C} / 744 \mathrm{~mm}\right) .\end{array}$ \\
\hline$(9.60)$ & $2 b$ & $\begin{array}{l}1.36 \\
(13.8)\end{array}$ & & s.Tab. 1 & \\
\hline $\begin{array}{l}1.68 \\
(10.0)\end{array}$ & 2b & $\begin{array}{l}1.40 \\
(14.2)\end{array}$ & 15 & s.Tab. 1 & unter sauerstoff. \\
\hline $\begin{array}{l}0.84 \\
(4.9)\end{array}$ & 2b & $\begin{array}{l}0.70 \\
(7.1)\end{array}$ & 15 & s.Tab. 1 & mit Krone-6 $6^{d}$ ). \\
\hline $\begin{array}{l}11.49 \\
(8.8) \\
1.27 \\
(7.5)\end{array}$ & $\begin{array}{l}2 c^{\prime} \\
2 c^{\prime}\end{array}$ & $\begin{array}{l}1.06 \\
(11.0) \\
1.05\end{array}$ & $\begin{array}{l}25 \\
20\end{array}$ & $\begin{array}{rl}0.01 & 3 c \\
& 0.005 \\
0.29 & 3 c \\
& -\end{array}$ & $\begin{array}{l}\text { 3c: gelbes } 010), \\
\left.+4(0.14 \mathrm{~g})^{f}\right) . \\
+4(0.07 \mathrm{~g}) \\
+5 a(0.27 \mathrm{~g}) \mathrm{g})\end{array}$ \\
\hline $\begin{array}{l}1.95 \\
(11.5)\end{array}$ & 2c & $\begin{array}{l}1.30 \\
(11.6)\end{array}$ & 15 & s. Tab. 1 & \\
\hline $\begin{array}{l}0.87 \\
(5.1)\end{array}$ & 2c & $\begin{array}{l}0.58 \\
(5.2)\end{array}$ & 15 & S.Tab. 1 & Zutropfen von $2 c^{h}$ ) \\
\hline $\begin{array}{l}0.75 \\
(4.4)\end{array}$ & 2c & $\begin{array}{l}0.50 \\
(4.5)\end{array}$ & 15 & S.Tab. 1 & \\
\hline $\begin{array}{l}0.63 \\
(3.7)\end{array}$ & $2 c$ & $\begin{array}{l}0.36 \\
(3.2)\end{array}$ & 10 & $0.183 \mathrm{c}$ & \\
\hline$(7.0)$ & $2 c$ & $\begin{array}{l}1.59 \\
(14.2)\end{array}$ & 20 & $0.073 \mathrm{c}$ & $\begin{array}{l}+5 \mathbf{a}(0.07 \mathbf{g}) \\
\left.+5 \mathrm{c}(0.32)^{i}\right)\end{array}$ \\
\hline & 2c & $\begin{array}{l}0.80 \\
(7.1)\end{array}$ & 15 & S.Tab. 1 & $\begin{array}{l}\text { mit } 18-\text { Krone-6 } \\
{[1.40 \mathrm{~g}(5.3 \mathrm{mmol})]}\end{array}$ \\
\hline 0.84 & $2 c$ & $\begin{array}{l}0.62 \\
(5.5)\end{array}$ & 15 & S.Tab. 1 & $\begin{array}{l}\text { mit } 18 \text {-krone-6 } \\
{[1.32 \mathrm{~g}(5.0 \mathrm{mmo} 1)]}\end{array}$ \\
\hline 0.84 & 20 & $\begin{array}{l}0.73 \\
(5.0)\end{array}$ & 10 & s. Tab. 1 & $\begin{array}{l}\text { Reaktion war nach } \\
4 \mathrm{~h} \text { beendet. }\end{array}$ \\
\hline 0.84 & 2d & $\begin{array}{l}1.46 \\
(10.0)\end{array}$ & 10 & $\begin{array}{l}0.11 \text { 3d } \\
0.54\end{array}$ & $\begin{array}{l}\text { 3d: Schmp. } 55-57^{\circ} \mathrm{C} \\
\left.\text { (Lit.54) } 55^{\circ} \mathrm{C}\right) .\end{array}$ \\
\hline 1.68 & 2d & $\begin{array}{l}1.50 \\
(10.2)\end{array}$ & 15 & & \\
\hline
\end{tabular}

:1 \%-Ausb., Reaktionszeit und -temp. siehe Tab. 1. - by Schmp. $37.5-38.5^{\circ} \mathrm{C}$ (Lit. $\left.\left.{ }^{48)} 39^{\circ} \mathrm{C}\right) .-{ }^{\circ}\right) \mathrm{C}_{9} \mathrm{H}_{11} \mathrm{NO}_{3}(181.2)$ Ber. C $59.66 \mathrm{H}$ 6.12 N 7.73. Gef. C $59.93 \mathrm{H} 6.14 \mathrm{~N} \mathrm{7.73.}-{ }^{3} \mathrm{H}-\mathrm{NMR}\left(\mathrm{CDCl}_{3}\right): \delta=$ $7.9-7.1\left(\mathrm{~m}, 4 \mathrm{H}, \mathrm{H}_{\text {arom }}\right), 4.65$ [hept $\left.J=7 \mathrm{~Hz}, 1 \mathrm{H}, \mathrm{CH}\left(\mathrm{CH}_{3}\right)_{2}\right], 1.4$ $\left[\mathrm{d}, J=7 \mathrm{~Hz}, 6 \mathrm{H}, \mathrm{CH}\left(\mathrm{CH}_{3}\right)_{2}\right] .-$ d) $1.32 \mathrm{~g}(5.0 \mathrm{mmol})$, der zusammen mit $2 \mathrm{~b}$ in $10 \mathrm{ml} \mathrm{TMH} \mathrm{zu} \mathrm{der} \mathrm{Lösung} \mathrm{von} 1$ in $5 \mathrm{ml} \mathrm{TMH}$ zugetropft wurde - e) ${ }^{1} \mathrm{H}-\mathrm{NMR}$ identisch mit Lit. ${ }^{50)}$ - Schmp. $97^{-9} 8^{\circ} \mathrm{C}$ (Lit. ${ }^{51)} 97^{\circ} \mathrm{C}$ ). - g) Schmp. $108-111^{\circ} \mathrm{C}$ (Lit. ${ }^{52)}$ $115.6^{\circ} \mathrm{C}$ ). - ${ }^{\text {h) }}$ Dann Rühren, $30 \mathrm{~min}$ bei $0^{\circ} \mathrm{C}$ und $24 \mathrm{~h}$ bei $50^{\circ} \mathrm{C}$. i) Schmp. $64-66^{\circ} \mathrm{C}$ (nach chromatographischer Aufarbeitung und MPLC der 1. Fraktion mit PE/EE $(9: 1)\left(\right.$ Lit. $\left.^{53)} 64.5^{\circ} \mathrm{C}\right)$. wurde nach dem Zusammengeben der Edukte Sauerstoff durch das Reaktionsgemisch geleitet (Tab. 7).

II) Umsetzungen meta-substituierter Nitrobenzole 6 mit Benzylalkohol/Kaliumhydroxid (zu Tab. 2). - Allgemeines: Frisch pulverisiertes Kaliumhydroxid, TBB und 6 wurden in einem $50-\mathrm{ml}-Z$ weihalskolben (mit Rührmagnet, Gummiseptum, Rückflußkühler und Gasblasenzähler) eingewogen und unter Sauerstoff nach Zuspritzen von Benzylalkohol in TMH (innerhalb $1 \mathrm{~h}$ ) gerührt. Aufarbeitung: a) Das Reaktionsgemisch wurde mit verd. $\mathrm{HCl}$ angesäuert, der Niederschlag abgesaugt, im Exsikkator über Phosphorpentoxid getrocknet und aus Methanol oder Ethanol umkristallisiert. b) Das Reaktionsgemisch wurde auf Eis gegossen, $2 \mathrm{mal}$ mit jeweils $50 \mathrm{ml}$ Diethylether ausgeschüttelt, der Extrakt mit Magnesiumsulfat getrocknet, im Rotationsverdampfer eingeengt und der Rückstand mit PE/EE (4:1) an Kieselgel chromatographiert. Nach Einengen des Eluats wurde der Rückstand aus Methanol oder Ethanol umkristallisiert.

Umsetzung von 3-Fluornitrobenzol (6a): Aus $1.41 \mathrm{~g}(10.0 \mathrm{mmol})$ 6a, $1.00 \mathrm{~g}(17.8 \mathrm{mmol})$ Kaliumhydroxid, $0.32 \mathrm{~g}(1.0 \mathrm{mmol}) \mathrm{TBB}$ und $1.30 \mathrm{~g}(12.0 \mathrm{mmol})$ Benzylalkohol in $10 \mathrm{ml} \mathrm{TMH}$. Nach Rühren ( $5 \mathrm{~h}$ bei Raumtemp. und $3 \mathrm{~h}$ bei $50^{\circ} \mathrm{C}$ ) und Aufarbeiten nach b);

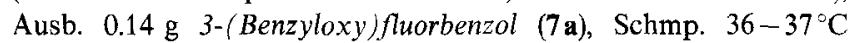
$(\mathrm{MeOH})\left(\right.$ Lit. $\left.^{55)} 42-43^{\circ} \mathrm{C}\right)$ und $\left.2.00 \mathrm{~g} \mathrm{3-(Benzyloxy}\right)$ nitrobenzol (3d), Schmp. $57-59^{\circ} \mathrm{C}\left(\right.$ EtOH) $\left(\right.$ Lit. $\left.^{54)} 55^{\circ} \mathrm{C}\right)$.

$$
\text { 7a: } \mathrm{C}_{13} \mathrm{H}_{11} \mathrm{FO}(202.2) \quad \text { Ber. C 77.21 H } 5.48 \text { F } 9.39
$$$$
\text { Gef. C } 77.27 \text { H } 5.54 \text { F } 8.64
$$

Umsetzung von 3-Chlornitrobenzol (6b): Aus $1.57 \mathrm{~g}(10.0 \mathrm{mmol})$ 6b, $1.00 \mathrm{~g}$ Kaliumhydroxid, $0.32 \mathrm{~g}$ TBB und $1.50 \mathrm{~g}(14.0 \mathrm{mmol})$ Benzylalkohol in $10 \mathrm{ml}$ TMH nach $24 \mathrm{stdg}$. Rühren bei $50^{\circ} \mathrm{C}$ und

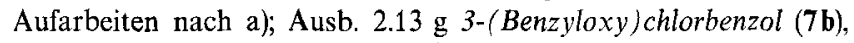
Schmp. $62-64^{\circ} \mathrm{C}(\mathrm{MeOH})\left[\mathrm{Lit}^{48)} 58^{\circ} \mathrm{C}(\mathrm{EtOH})\right]$.

Umsetzung von 3-Bromnitrobenzol (6c): Aus $2.02 \mathrm{~g}$ (10.0 mmol) $6 \mathrm{c}, 1.00 \mathrm{~g}$ Kaliumhydroxid, $0.32 \mathrm{~g}$ TBB und $1.50 \mathrm{~g}(14.0 \mathrm{mmol})$ Benzylalkohol in $10 \mathrm{ml} \mathrm{TMH}$ nach $24 \mathrm{stdg}$. Rühren bei $50^{\circ} \mathrm{C}$ und Aufarbeiten nach a); Ausb. 2.47 g 3-(Benzyloxy)brombenzol (7c), Schmp. $63-64^{\circ} \mathrm{C}(\mathrm{MeOH})\left[\right.$ Lit. $\left.^{56)} 59-63^{\circ} \mathrm{C}(\mathrm{MeOH})\right]$.

Umsetzung von 3-Iodnitrobenzol (6d): $2.49 \mathrm{~g}(10.0 \mathrm{mmol}) \mathbf{6 d}$, $1.00 \mathrm{~g}$ Kaliumhydroxid, $0.32 \mathrm{~g}$ TBB und $1.88 \mathrm{~g}$ (17.4 mmol) Benzylalkohol in $10 \mathrm{ml} \mathrm{TMH}$ wurden $40 \mathrm{~h}$ bei $50^{\circ} \mathrm{C}$ gerührt und nach b) aufgearbeitet; nach $\mathrm{GC}$ war keine vollständige Umsetzung von 6d eingetreten, die Reaktionsprodukte wurden mittels GC/MS (70 eV) bestimmt.

3-(Benzyloxy)iodbenzol (7d): MS: $m / z(\%)=311(29)\left[\mathrm{M}^{+}+1\right]$, $91(100)\left[\mathrm{C}_{7} \mathrm{H}_{7}^{+}\right]$.

3,3'-Diiodazoxybenzol: MS: $m / z(\%)=451(38)\left[\mathrm{M}^{+}+1\right], 435$ (43) $\left[\mathrm{M}^{+}+1-\mathrm{O}\right], 324(21)\left[\mathrm{M}^{+}+1-\mathrm{I}\right], 308(43)\left[\mathrm{M}^{+}+1-\right.$ I $-\mathrm{O}$ ].

Nitrobenzol: MS: $m / z(\%)=124(100)\left[\mathrm{M}^{+}+1\right], 108(8)\left[\mathrm{M}^{+}+\right.$ 1 - O], 94 (7) $\left[\mathrm{M}^{+}+1-\mathrm{NO}\right), 78(4)\left[\mathrm{M}^{+}+1-\mathrm{NO}_{2}\right]$.

Umsetzung von 3-Nitro(phenylsulfonyl)benzol (6e): Aus $2.60 \mathrm{~g}$ (10.0 mmol) 6e, $1.00 \mathrm{~g}$ Kaliumhydroxid, $0.32 \mathrm{~g}$ TBB und $1.35 \mathrm{~g}$ (12.5 mmol) Benzylalkohol in $10 \mathrm{ml}$ TMH nach 8stdg. Rühren bei Raum-

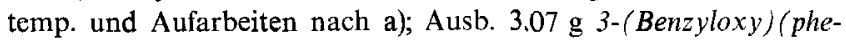
nylsulfonyl)benzol (7e), Schmp. 98-99 $\mathrm{C}$ (Ethanol). - ${ }^{1} \mathrm{H}-\mathrm{NMR}$ $\left(\mathrm{CDCl}_{3}\right): \delta=5.13\left(\mathrm{~s}, 2 \mathrm{H}, \mathrm{CH}_{2}\right), 7.10-7.67$ und $7.80-8.07(\mathrm{~m}, 14 \mathrm{H}$, $\mathbf{H}_{\text {arom. }}$ ).

$\mathrm{C}_{19} \mathrm{H}_{16} \mathrm{O}_{3} \mathrm{~S}$ (324.4) Ber. C $70.35 \mathrm{H} 4.97 \mathrm{~S} 9.88$ Gef. C 70.16 H 4.99 S 10.07

Umsetzung von 3-Nitrobenzonitril (6f): $1.48 \mathrm{~g} \mathrm{(10.0} \mathrm{mmol)} 6 \mathrm{f}$, $0.80 \mathrm{~g}$ (14.3 mmol) Kaliumhydroxid, $0.32 \mathrm{~g}$ TBB und $1.30 \mathrm{~g}$ (12.0 
mmol) Benzylalkohol in $10 \mathrm{ml} \mathrm{TMH}$ wurden $20 \mathrm{~h}$ bei Raumtemp. gerührt und nach b) aufgearbeitet. Das erhaltene Eluat wurde mittels MPLC [PE/EE (9:1)] getrennt; Ausb. $0.31 \mathrm{~g}$ 3-(Benzyloxy)benzonitril (7f), Schmp. $39-40^{\circ} \mathrm{C}$ (Lit. ${ }^{57)} 40-41^{\circ} \mathrm{C}$ ).

III) Umsetzungen von 1,3,5-trisubstituierten Benzolen 9 und 10 mit 2d (zu Tab. 3). - Allgemeines: Umsetzung und Aufarbeitung siehe unter II.

Umsetzungen von 1,3,5-Trinitrobenzal (9a) und 5-(Benzyloxy)1,3-dinitrobenzol (10 a): a) Aus $2.13 \mathrm{~g}(10.0 \mathrm{mmol}) \mathbf{9 a}, 1.85 \mathrm{~g}(33.0$ mmol) Kaliumhydroxid, $0.32 \mathrm{~g}$ TBB und $1.30 \mathrm{~g}(12.0 \mathrm{mmol})$ Benzylalkohol in $10 \mathrm{ml}$ TMH nach Rühren ( $48 \mathrm{~h}$ bei Raumtemp. und $12 \mathrm{~h}$ bei $50^{\circ} \mathrm{C}$ ) und Aufarbeiten nach b); Ausb. $0.38 \mathrm{~g} \mathrm{10a}$, Schmp. $148-150^{\circ} \mathrm{C}(\mathrm{EtOH}) .-{ }^{\mathrm{t}} \mathrm{H}-\mathrm{NMR}\left(\mathrm{CDCl}_{3}\right): \delta=5.25(\mathrm{~s}, 2 \mathrm{H}$, $\left.\mathrm{CH}_{2} \mathrm{C}_{6} \mathrm{H}_{5}\right), 7.39-7.46\left(\mathrm{~m}, 5 \mathrm{H}, \mathrm{C}_{6} H_{5} \mathrm{CH}_{2}\right), 8.13(\mathrm{~d}, J=1.96 \mathrm{~Hz}, 2 \mathrm{H}$, $\left.\mathrm{H}_{\text {arom }}\right), 9.64\left(\mathrm{t}, 1 \mathrm{H}, \mathrm{H}_{\text {arom }}\right)$.

$$
\begin{array}{lll}
\mathrm{C}_{13} \mathrm{H}_{10} \mathrm{~N}_{2} \mathrm{O}_{5} \text { (274.2) } & \text { Ber. C } 56.94 \mathrm{H} 3.67 \mathrm{~N} 10.21 \\
& \text { Gef. C } 57.01 \text { H } 3.71 \text { N } 10.26
\end{array}
$$

b) $2.13 \mathrm{~g} \mathrm{9a}, 4.00 \mathrm{~g}(28.9 \mathrm{mmol})$ Kaliumcarbonat, $0.32 \mathrm{~g}$ TBB und $1.49 \mathrm{~g}(13.8 \mathrm{mmol})$ Benzylalkohol in $10 \mathrm{ml} \mathrm{TMH}$ wurden $24 \mathrm{~h}$ bei $50^{\circ} \mathrm{C}$ und $21 \mathrm{~h}$ bei $80^{\circ} \mathrm{C}$ gerührt und nach b) aufgearbeitet. Der erhaltene Rückstand wurde mit Dichlormethan an Kieselgel chromatographiert; Ausb. $1.95 \mathrm{~g} \mathrm{10a}$.

c) Aus $2.74 \mathrm{~g}(10.0 \mathrm{mmol}) 10 \mathrm{a}, 1.00 \mathrm{~g}$ Kaliumhydroxid, $0.32 \mathrm{~g}$ TBB und $1.30 \mathrm{~g}$ Benzylalkohol in $10 \mathrm{ml} \mathrm{TMH}$ nach 5stdg. Rühren

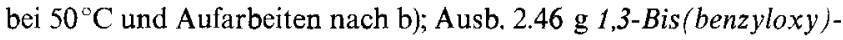
5-nitrobenzol (11a), Schmp. 107-110 ${ }^{\circ} \mathrm{C}$ (EtOH). - ' $\mathrm{H}-\mathrm{NMR}$ $\left(\mathrm{CDCl}_{3}\right): \delta=5.10\left(\mathrm{~s}, 4 \mathrm{H}, 2 \mathrm{CH}_{2} \mathrm{C}_{6} \mathrm{H}_{5}\right), 6.90\left(\mathrm{t}, J=2 \mathrm{~Hz}, 1 \mathrm{H}, \mathrm{H}_{\text {arom }}\right)$, $7.30-7.50\left(\mathrm{~m}, 10 \mathrm{H}, 2 \mathrm{CH}_{2} \mathrm{C}_{6} \mathrm{H}_{5}\right)$.

$$
\begin{array}{ll}
\mathrm{C}_{20} \mathrm{H}_{17} \mathrm{NO}_{4} \text { (335.4) } & \text { Ber. C } 71.63 \mathrm{H} 5.11 \mathrm{~N} 4.18 \\
& \text { Gef. C } 71.80 \mathrm{H} 5.10 \mathrm{~N} 4.15
\end{array}
$$

Umsetzung von 1-Chlor-3,5-dinitrobenzol (9b) und 1-(Benzyloxy)3-chlor-5-nitrobenzol (10b): a) $2.16 \mathrm{~g}(10.7 \mathrm{mmol}) 9 \mathrm{~b}, 1.00 \mathrm{~g}$ (17.8 mmol) Kaliumhydroxid, $0.32 \mathrm{~g}$ TBB und $1.30 \mathrm{~g}$ Benzylalkohol in $10 \mathrm{ml}$ TMH wurden $6 \mathrm{~h}$ bei Raumtemp. gerührt und nach b) aufgcarbeitet; Ausb. $2.06 \mathrm{~g} 10 \mathrm{~b}, \mathrm{Schmp} .53-55^{\circ} \mathrm{C}(\mathrm{EtOH}),-{ }^{1} \mathrm{H}-$ NMR $\left(\mathrm{CDCl}_{3}\right): \delta=5.18\left(\mathrm{~s}, 2 \mathrm{H}, \mathrm{CH}_{2} \mathrm{C}_{6} \mathrm{H}_{5}\right), 7.25-7.40(\mathrm{~m}, 1 \mathrm{H}$, $\left.\mathrm{H}_{\text {arom }}\right), 7.45\left(\mathrm{~s}, 5 \mathrm{H}, \mathrm{CH}_{2} \mathrm{C}_{6} \mathrm{H}_{5}\right), 7.65-7.90\left(\mathrm{~m}, 2 \mathrm{H}, \mathrm{H}_{\text {arom }}\right)$.

$$
\begin{aligned}
& \mathrm{C}_{13} \mathrm{H}_{10} \mathrm{ClNO}_{3}(263.7) \\
& \text { Ber. C } 59.22 \mathrm{H} 3.82 \mathrm{Cl} 13.44 \text { N } 5.31 \\
& \text { Gef. C } 59.47 \text { H } 3.83 \text { Cl } 13.36 \text { N } 5.30
\end{aligned}
$$

b) $1.32 \mathrm{~g}(5.02 \mathrm{mmol}) \mathbf{1 0 b}, 0.50 \mathrm{~g}(8.9 \mathrm{mmol})$ Kaliumhydroxid, $0.16 \mathrm{~g}(0.5 \mathrm{mmol}) \mathrm{TBB}$ und $0.84 \mathrm{~g}(7.8 \mathrm{mmol})$ Benzylalkohol in $5 \mathrm{ml}$ TMH wurden $24 \mathrm{~h}$ bei $50^{\circ} \mathrm{C}$ gerührt und nach a) aufgearbeitet; Ausb. $1.49 \mathrm{~g} \mathrm{1,3-Bis(benzyloxy)-5-chlorbenzol}$ (11 b), Schmp. $65-66{ }^{\circ} \mathrm{C}(\mathrm{MeOH}),-{ }^{1} \mathrm{H}-\mathrm{NMR}\left(\mathrm{CDCl}_{3}\right): \delta=4.93(\mathrm{~s}, 4 \mathrm{H}$, $\left.2 \mathrm{CH}_{2} \mathrm{C}_{6} \mathrm{H}_{5}\right), 6.41-6.70\left(\mathrm{~m}, 3 \mathrm{H}, \mathrm{H}_{\text {arom }}\right), 7.39$ (s, $\left.10 \mathrm{H}, 2 \mathrm{CH}_{2} \mathrm{C}_{6} \mathrm{H}_{5}\right)$.

$$
\begin{array}{ll}
\mathrm{C}_{20} \mathrm{H}_{17} \mathrm{ClO}_{2} \text { (324.8) } & \text { Ber. C 73.96 H } 5.27 \mathrm{Cl} 10.91 \\
& \text { Gef. C 74.00 H } 5.34 \mathrm{Cl} 10.92
\end{array}
$$

Umsetzung von 1-Brom-3,5-dinitrobenzol (9c) und 1-(Benzyloxy)3-chlor-5-nitrobenzol (10c): a) $2.47 \mathrm{~g}$ (10.0 mmol) 9c, $1.00 \mathrm{~g} \mathrm{Kalium-}$ hydroxid, $0.32 \mathrm{~g} \mathrm{TBB}$ und $1.24 \mathrm{~g}(11.5 \mathrm{mmol})$ Benzylalkohol in $10 \mathrm{ml}$ TMH wurden $8 \mathrm{~h}$ bei Raumtemp. gerührt und nach b) aufgearbeitet; Ausb. $2.27 \mathrm{~g} \mathrm{10c}$, Schmp. $59-61{ }^{\circ} \mathrm{C}(\mathrm{MeOH}) .-{ }^{1} \mathrm{H}$ NMR $\left(\mathrm{CDCl}_{3}\right): \delta=5.10\left(\mathrm{~s}, 2 \mathrm{H}, \mathrm{CH}_{2} \mathrm{C}_{6} \mathrm{H}_{5}\right), 7.30-7.47(\mathrm{~m}, 5 \mathrm{H}$, $\left.\mathrm{CH}_{2} \mathrm{C}_{6} H_{5}\right), 7.73\left(\mathrm{t}, J=2 \mathrm{~Hz}, 1 \mathrm{H}, \mathrm{H}_{\text {arom }}\right), 7.95\left(\mathrm{t}, 1 \mathrm{H}, \mathrm{H}_{\text {arom }}\right)$.

$\mathrm{C}_{13} \mathrm{H}_{10} \mathrm{BrNO}_{3}$ (308.1)

Ber. C 50.67 H 3.27 Br 25.93 N 4.55

Gef. C 50.77 H 3.33 Br 26.13 N 4.55

b) $3.08 \mathrm{~g}$ (10.0 mmol) $10 \mathrm{c}, 1.00 \mathrm{~g}$ Kaliumhydroxid, $0.32 \mathrm{~g}$ TBB und $1.50 \mathrm{~g}$ Benzylalkohol in $10 \mathrm{ml} \mathrm{TMH}$ wurden $24 \mathrm{~h}$ bei $50^{\circ} \mathrm{C}$ gerührt und nach a) aufgearbeitet; Ausb. $3.16 \mathrm{~g} \mathrm{1,3-Bis(benzyloxy)-}$ 5-brombenzol (11 c), Schmp. $65-67^{\circ} \mathrm{C}(\mathrm{MeOH})\left(\mathrm{Lit}^{58)} 66^{\circ} \mathrm{C}\right)$.

Umsetzung von 1,3-Dichlor-5-nitrobenzol (9d): Aus $1.92 \mathrm{~g}(10.0$ mmol) 9d, $1.00 \mathrm{~g}$ Kaliumhydroxid, $0.32 \mathrm{~g}$ TBB und $1.30 \mathrm{~g}$ Benzylalkohol in $10 \mathrm{ml}$ TMH nach 25stdg. Rühren bei Raumtemp. und Aufarbeiten nach b); Ausb. $2.35 \mathrm{~g} 1$-(Benzyloxy)-3,5-dichlorbenzol (10d), Schmp. $21^{\circ} \mathrm{C}$. $-{ }^{1} \mathrm{H}-\mathrm{NMR}\left(\mathrm{CDCl}_{3}\right): \delta=4.96(\mathrm{~s}, 2 \mathrm{H}$, $\left.\mathrm{CH}_{2} \mathrm{C}_{6} \mathrm{H}_{5}\right), 6.80-7.00\left(\mathrm{~m}, 3 \mathrm{H}, \mathrm{H}_{\text {arom }}\right), 7.40\left(\mathrm{~s}, 5 \mathrm{H}, \mathrm{CH}_{2} \mathrm{C}_{6} \mathrm{H}_{5}\right)$.

$$
\mathrm{C}_{13} \mathrm{H}_{10} \mathrm{Cl}_{2} \mathrm{O}(253.1) \quad \text { Ber. } \mathrm{C} 61.68 \mathrm{H} 3.98 \mathrm{Cl} 28.01
$$

Gef. C 61.72 H 4.21 Cl 27.87

Umsetzung von 1,3-Dibrom-5-nitrobenzol (9e): $2.81 \mathrm{~g}(10.0 \mathrm{mmol})$ 9e, $1.00 \mathrm{~g}$ Kaliumbydroxid, $0.32 \mathrm{~g}$ TBB und $1.30 \mathrm{~g}$ Benzylalkohol in $10 \mathrm{ml} \mathrm{TMH}$ wurden $7.5 \mathrm{~h}$ bei Raumtemp. gerührt und nach b) aufgearbeitet. Das Eluat wurde bei $128-130^{\circ} \mathrm{C} / 10^{-3}$ Torr destilliert; Ausb. 3.05 g 1-(Benzyloxy)-3,5-dibrombenzol (10e). $-{ }^{1} \mathrm{H}$ NMR $\left(\mathrm{CDCl}_{3}\right): \delta=4.95\left(\mathrm{~s}, 2 \mathrm{H}, \mathrm{CH}_{2} \mathrm{C}_{6} \mathrm{H}_{5}\right), 7.03(\mathrm{~d}, J=2 \mathrm{~Hz}, 2 \mathrm{H}$, $\left.\mathrm{H}_{\text {arom. }}\right), 7.23\left(\mathrm{t}, 1 \mathrm{H}, \mathrm{H}_{\text {arom }}\right), 7.35\left(\mathrm{~s}, 5 \mathrm{H}, \mathrm{CH}_{2} \mathrm{C}_{6} \mathrm{H}_{5}\right)$.

$$
\begin{array}{ll}
\mathrm{C}_{13} \mathrm{H}_{10} \mathrm{Br}_{2} \mathrm{O} \text { (342.0) } & \text { Ber. C } 45.65 \mathrm{H} 2.95 \mathrm{Br} 46.72 \\
& \text { Gef. C } 45.82 \mathrm{H} 3.02 \mathrm{Br} 46.70
\end{array}
$$

IV) Umsetzungen von 4,4'-Dinitrobiphenyl (12) mit Alkoholaten 2 , Natriumalkanthiolaten $14 \mathbf{a}^{\prime}, \mathbf{f}^{\prime}$ und Kaliumthiophenolat (14e) in TMH (zu Tab. 4). - a) Umsetzungen mit Alkoholaten 2. - Allgemeines: Die Edukte $[1.00 \mathrm{~g}(4.1 \mathrm{mmol}) 12$ und $12.5 \mathrm{mmol}$ 2] wurden in einem ausgeheizten, mit Stickstoff belüfteten Kolben unter Stickstoff eingewogen; danach wurde das Gefäß mit einem Gummiseptum verschlossen, TMH $(14 \mathrm{ml})$ zugespritzt und unter Stickstoff umgesetzt. Nach Reaktionsende wurde mit $0.1 \mathrm{~N} \mathrm{HCl}$ angesäuert, das ausgefallene Produkt abgesaugt, über Phosphorpentoxid getrocknet und aus Ethanol umkristallisiert.

4'-Methoxy-4-nitrobiphenyl (13a): Aus 12 und $0.66 \mathrm{~g}$ Natriummethanolat (2a') in TMH nach 1stdg. Rühren bei Raumtemp. und Aufarbeiten; Ausb. 0.92 g, Schmp. $110.5^{\circ} \mathrm{C}$ (Lit. ${ }^{40 a)} 111^{\circ} \mathrm{C}$ ).

4'-(Benzyloxy)-4-nitrobiphenyl (13d): Aus 12 und $1.59 \mathrm{~g}$ Natriumbenzylalkoholat (2d') in TMH nach 2 stdg. Rühren bei Raumtemp. und Aufarbeiten; Ausb. $1.18 \mathrm{~g}$, Schmp. $171^{\circ} \mathrm{C}$ (Lit. ${ }^{59)} 171-172^{\circ} \mathrm{C}$ ).

4'-Phenoxy-4-nitrobiphenyl (13e): Aus 12 und 1.62 g Kaliumphenolat (2e) in TMH nach 72stdg. Rühren bei $70^{\circ} \mathrm{C}$ und Aufarbeiten; Ausb. $1.11 \mathrm{~g}$, Schmp. $117-118^{\circ} \mathrm{C} .-{ }^{1} \mathrm{H}-\mathrm{NMR}\left(\mathrm{CDCl}_{3}\right): \delta=$

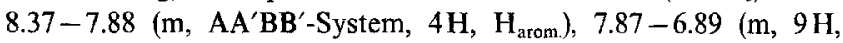
$\mathrm{H}_{\text {arom. }}$ ). $\mathrm{C}_{18} \mathrm{H}_{13} \mathrm{NO}_{3}(291.3)$ Ber. C 74.22 H $4.48 \mathrm{~N} 4.81$ Gef. C 73.74 H 4.67 N 4.91

b) Umsetzungen mit Natriummethan-(14a'), -ethanthiolat (14f') und Kaliumthiophenolat (14e):

4'-(Methylthio)-4-nitrobiphenyl (15a): $0.50 \mathrm{~g}$ (2.1 mmol) 12 wurde wie unter a) beschrieben unter Stickstoff eingewogen; anschließend wurden $30 \mathrm{ml}$ TMH zugespritzt und $0.30 \mathrm{~g}(4.3 \mathrm{mmol}) 14 \mathrm{a}^{\prime}$ aus einer Substanzbirne bei $-2^{\circ} \mathrm{C}$ zugegeben. Nach 6 stdg. Rühren bei $-2^{\circ} \mathrm{C}$ unter Stickstoff wurde das Produkt durch Ansäuern mit $0.1 \mathrm{~N} \mathrm{HCl}$ ausgefällt, nach 15 min abgesaugt und in Dichlormethan gelöst. Nach Trocknen mit Calciumchlorid wurde im Rotationsverdampfer eingeengt, der Rückstand in Dichlormethan/Ethylacetat (2:1) gelöst und an basischem Aluminiumoxid chromatographiert. Das Eluat wurde im Rotationsverdampfer eingeengt und der Rückstand i.Vak. (Ölpumpe) getrocknet; Ausb. $0.43 \mathrm{~g}$, Schmp. $138.5^{\circ} \mathrm{C} .-{ }^{1} \mathrm{H}-\mathrm{NMR}\left(\mathrm{CDCl}_{3}\right): \delta=8.30-7.69\left(\mathrm{~m}, \mathrm{AA}^{\prime} \mathrm{BB}^{\prime}\right.$-System, $\left.4 \mathrm{H}, \mathrm{H}_{\text {arom }}\right), 7.57-7.34\left(\mathrm{~m}, \mathrm{AA}^{\prime} \mathbf{B B}^{\prime}-\right.$ System, $\left.4 \mathrm{H}, \mathrm{H}_{\text {arom }}\right), 2.54(\mathrm{~s}, 3 \mathrm{H}$, $\mathrm{SCH}_{3}$ ).

$\mathrm{C}_{13} \mathrm{H}_{11} \mathrm{NO}_{2} \mathrm{~S}$ (245.3)

Ber. C 63.66 H 4.52 N 5.71 S 13.07

Gef. C 63.46 H 4.43 N 5.74 S 12.98 
$4^{\prime}$-(Ethylthio)-4-nitrobiphenyl (15f): Wie voranstehend beschrieben aus $0.50 \mathrm{~g}$ ( $2.1 \mathrm{mmol}) 12$ und $0.35 \mathrm{~g}(4.2 \mathrm{mmol}) 14 \mathrm{f}^{\prime}$ in $7 \mathrm{ml}$ TMH. Zur Aufarbeitung wurde nach Einengen der DichlormethanLösung der Rückstand mit tiefsiedendem Petrolether in einer Soxhlet-Apparatur fraktionierend extrahiert und nach Einengen des Extrakts der Rückstand i.Vak. (Ölpumpe) getrocknet; Ausb. $0.38 \mathrm{~g}$, Schmp. $87^{\circ} \mathrm{C}$. $-{ }^{1} \mathrm{H}-\mathrm{NMR}\left(\mathrm{CDCl}_{3}\right): \delta=8.31-7.79\left(\mathrm{~m}, \mathrm{AA}^{\prime} \mathrm{BB}^{\prime}-\right.$ System, 4H, $\mathbf{H}_{\text {arom }}$ ), 7.56-7.39 (m, $\mathrm{AA}^{\prime} \mathrm{BB}^{\prime}$-System, 4H, $\mathrm{H}_{\text {arom }}$ ), $3.05-2.98\left(\mathrm{q}, 2 \mathrm{H}, \mathrm{SCH}_{2} \mathrm{CH}_{3}\right), 1.39-1.35\left(\mathrm{t}, 3 \mathrm{H}, \mathrm{SCH}_{2} \mathrm{CH}_{3}\right)$.

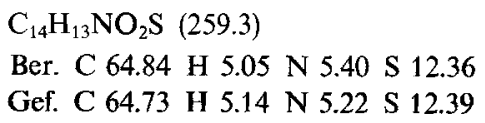

4'-(Phenylthio)-4-nitrobiphenyl (15e): $12.00 \mathrm{~g}$ (4.1 mmol) 12 und $1.22 \mathrm{~g}(8.6 \mathrm{mmol}) 14 \mathrm{e}$ in $14 \mathrm{ml} \mathrm{TMH}$ wurden $72 \mathrm{~h}$ bei $40^{\circ} \mathrm{C}$ gerührt und wie voranstehend beschrieben aufgearbeitet. Die Dichlormethan-Lösung wurde eingeengt, der Rückstand erneut in Dichlormethan gelöst, an basischem Aluminiumoxid mit Dichlormethan/ Ethylacetat eluiert und das Eluat eingeengt; Ausb. $0.88 \mathrm{~g}$, Schmp. $102^{\circ} \mathrm{C}$. $-{ }^{1} \mathrm{H}-\mathrm{NMR}\left(\mathrm{CDCl}_{3}\right): \delta=8.31-7.69$ (m, AA'BB'-System, $\left.4 \mathrm{H}, \mathrm{H}_{\text {arom }}\right), 7.55-7.32\left(\mathrm{~m}, 9 \mathrm{H}, \mathrm{H}_{\text {arom }}\right)$.

\section{$\mathrm{C}_{18} \mathrm{H}_{13} \mathrm{NO}_{2} \mathrm{~S}$ (307.4) \\ Ber. C 70.34 H 4.26 N $4.56 \mathrm{~S} 10.34$ \\ Gef. C 70.19 H 4.25 N 4.73 S 10.36}

V) Umsetzungen von stellungsisomeren Dinitrobiphenylen mit $\mathrm{Na}$ triummethanolat (2 $\left.\mathbf{a}^{\prime}\right)$ (zu Tab. 5). - Allgemeines: Umsetzungen und Aufarbeitung siehe unter IVa.

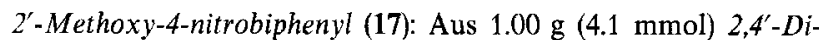
nitrobiphenyl (16) und $0.66 \mathrm{~g}(12.30 \mathrm{mmol}) 2 \mathrm{a}^{\prime}$ in $14 \mathrm{ml}$ TMH nach 3.5 h; Ausb. 0.85 g, Schmp. $51^{\circ} \mathrm{C}$ (Lit. ${ }^{60)} 50-51^{\circ} \mathrm{C}$ ).

2'-Methoxy-2-nitrobiphenyl (19): Aus $1.00 \mathrm{~g}$ (4.1 mmol) 2,2'-Dinitrobiphenyl (18) und $0.66 \mathrm{~g} 2 \mathrm{a}^{\prime}$ nach $21 \mathrm{~h}$; Ausb. $0.75 \mathrm{~g}$, Schmp. $80-81^{\circ} \mathrm{C}$ ( Lit. $^{61)} 80-81^{\circ} \mathrm{C}$ ).

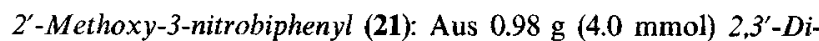
nitrobiphenyl (20) und $0.63 \mathrm{~g}(11.7 \mathrm{mmol}) 2 \mathrm{a}^{\prime}$ in $14 \mathrm{ml}$ TMH nach 4 h; Ausb. 0.72 g Öl, nach Reinigung mittels MPLC 0.64 g, Schmp. $48-49^{\circ} \mathrm{C}$ (Lit. ${ }^{62)} 66^{\circ} \mathrm{C}$; die nach Lit. $^{62)}$ dargestellte Verbindung zeigte ebenfalls einen Schmp. von $\left.47-50^{\circ} \mathrm{C}\right)$. - ${ }^{1} \mathrm{H}-\mathrm{NMR}\left(\mathrm{CDCl}_{3}\right)$; $\delta=8.42-8.41\left(\mathrm{t}, 1 \mathrm{H}, \mathrm{H}_{\text {arom }}\right), 8.18-8.15$ und $7.87-7.83(2 \mathrm{~m}, 1 \mathrm{H}$, $\mathrm{H}_{\text {arom. }}$ ), $7.58-7.52$ (t, $J=7.95 \mathrm{~Hz}, 1 \mathrm{H}, \mathrm{H}_{\text {arom }}$ ), $7.42-7.32$ und $7.09-7.00\left(2 \mathrm{~m}, 2 \mathrm{H}, \mathrm{H}_{\text {arom }}\right), 3.84\left(\mathrm{~s}, 3 \mathrm{H}, \mathrm{OCH}_{3}\right)$.

$$
\mathrm{C}_{13} \mathrm{H}_{41} \mathrm{NO}_{3} \text { (229.2) Ber. C } 68.11 \text { H } 4.84 \text { N } 6.11
$$

Gef. C 68.17 H 4.96 N 5.91

4'-Methoxy-3-nitro- (23) und 3'-Methoxy-4-nitrobiphenyl (24): Aus $1.00 \mathrm{~g}$ (4.1 mmol) 22 und $0.66 \mathrm{~g}$ (12.2 mmol) $2 \mathrm{a}^{\prime}$ nach $18 \mathrm{~h}$; Ausb. $0.78 \mathrm{~g}$ Gemisch aus 23/24 (7:1, Kapillar-GC), das mittels MPLC [PE/EE $(9: 1)]$ getrennt wurde.

23: Schmp. $79^{\circ} \mathrm{C}$ (Lit. ${ }^{63)} 82^{\circ} \mathrm{C}$ ).

24: Schmp. $90-91^{\circ} \mathrm{C}$ (Lit. ${ }^{64)}$ Isomerengemisch ohne Schmp.-Angabe). $-{ }^{1} \mathrm{H}$-NMR $\left(\mathrm{CDCl}_{3}\right): \delta=8.31-8.27$ und $7.75-7.70(2 \mathrm{~m}$, $\left.2 \mathrm{H}, \mathrm{H}_{\text {arom. }}\right), 7.44-7.39\left(\mathrm{t}, J=7.95 \mathrm{~Hz}, 1 \mathrm{H}, \mathrm{H}_{\mathrm{ph}}\right), 7.22-7.13(\mathrm{~m}$, $\left.2 \mathrm{H}, \mathrm{H}_{\mathrm{Ph}}\right), 7.01-7.00\left(\mathrm{~m}, 1 \mathrm{H}, \mathrm{H}_{\text {arom. }}\right), 3.88\left(\mathrm{~s}, 3 \mathrm{H}, \mathrm{CH}_{3} \mathrm{O}\right)$.

$$
\begin{aligned}
& \text { 24: } \mathrm{C}_{13} \mathrm{H}_{11} \mathrm{NO}_{3}(229.2) \text { Ber. C } 68.11 \mathrm{H} 4.84 \mathrm{~N} 6.11 \\
& \text { Gef. C } 68.08 \text { H } 4.96 \text { N } 5.96
\end{aligned}
$$

3'-Methoxy-3-nitrobiphenyl (26): Aus $1.00 \mathrm{~g}$ (4.1 mmol) 3,3'-Dinitrobiphenyl (25) und $0.66 \mathrm{~g}$ (12.2 $\mathrm{mmol}) 2 \mathrm{a}^{\prime}$ in $14 \mathrm{ml} \mathrm{TMH}$ nach $24 \mathrm{~h}$; Ausb. $0.27 \mathrm{~g}$, Schmp. $77-78^{\circ} \mathrm{C}$ (Methanol). - ${ }^{1} \mathrm{H}-\mathrm{NMR}$ $\left(\mathrm{CDCl}_{3}\right): \delta=8.45-8.44\left(\mathrm{t}, J=1.88 \mathrm{~Hz}, 1 \mathrm{H}, \mathrm{H}_{\text {arom. }}\right), 8.22-8.18$ und $7.92-8.89\left(2 \mathrm{~m}, 1 \mathrm{H}, \mathrm{H}_{\mathrm{arom}}\right), 7.63-7.58$ und $7.44-7.38(2 \mathrm{t}$,
$\left.J=7.98 \mathrm{~Hz}, 1 \mathrm{H}, \mathrm{H}_{\text {arom }}\right), 7.22-7.13\left(\mathrm{~m}, 2 \mathrm{H}_{\text {arom }}\right), 6.99-6.96(\mathrm{~m}$, $\left.1 \mathrm{H}, \mathrm{H}_{\text {arom. }}\right)$ und $3.89\left(\mathrm{~s}, 3 \mathrm{H}, \mathrm{OCH}_{3}\right)$.

$$
\begin{array}{ll}
\mathrm{C}_{13} \mathrm{H}_{11} \mathrm{NO}_{3} \text { (229.2) } & \text { Ber. C } 68.11 \text { H } 4.84 \text { N } 6.11 \\
& \text { Gef. C } 67.90 \text { H } 5.00 \text { N } 5.93
\end{array}
$$

Vergleichende Umsetzungen von Dinitrobiphenylen mit $\mathbf{2} \mathbf{a}^{\prime}$ in TMH bei Raumtemperatur (zu Tab. 6): Stammlösung: $1.00 \mathrm{~g} \mathrm{(4.1}$ mmol) Dinitrobiphenyl und $0.10-0.15 \mathrm{~g}$ Eicosan wurden unter Stickstoff in einem ausgeheizten Kolben eingewogen und mittels einer Spritze mit $20 \mathrm{ml} \mathrm{TMH} \mathrm{versetzt.} 4 \mathrm{ml}$ dieser Stammlösung wurden durch ein Gummiseptum zu $0.13 \mathrm{~g}(2.4 \mathrm{mmol}) 2 \mathrm{a}^{\prime}$ gespritzt; dann wurde das Gemisch i. Vak. entgast und mit Stickstoff belüftet. Mit einer Spritze wurde eine Probe entnommen, in Diethylether gegeben und der Eichfaktor mittels Kapillar-GC bestimmt. Nach ca. 3-5 min. Behandeln mit Ultraschall wurden nach $2 \mathrm{~h}$ und $20 \mathrm{~h}$ Stehenlassen Proben entnommen, die mit Diethylether verdünnt und dann filtriert wurden. Der Gehalt an noch vorhandenem Edukt wurde mittels Kapillar-GC bestimmt.

\section{VII) 4-Methoxybiphenyl (28)}

$2.03 \mathrm{~g}$ (10.2 mmol) 4-Nitrobiphenyl (27) wurden mit $1.22 \mathrm{~g}(8.24$ mmol) $2 \mathrm{a}$ in $35 \mathrm{ml}$ TMH wie unter IVa beschrieben $48 \mathrm{~h}$ bei Raumtemp. umgesetzt; Ausb. $1.50 \mathrm{~g}(81 \%)$, Schmp. $90^{\circ} \mathrm{C}$ (Lit. ${ }^{65)} 90^{\circ} \mathrm{C}$ ).

VIII) Umsetzungen von Nitronaphthalinen mit Benzylalkohol/Kaliumhydroxid. - Allgemeines: Umsetzungen und Aufarbeitung siehe unter II.

Umsetzung von 1,5-Dinitronaphthalin (29): Aus $2.18 \mathrm{~g}(10.0 \mathrm{mmol})$ 29, $1.00 \mathrm{~g}$ Kaliumhydroxid, $0.32 \mathrm{~g}$ TBB und $1.29 \mathrm{~g}(11.9 \mathrm{mmol})$ Benzylalkohol in $10 \mathrm{ml}$ TMH nach Rühren ( $4 \mathrm{~h}$ bei Raumtemp. und $1 \mathrm{~h}$ bei $\left.50^{\circ} \mathrm{C}\right)$ und Aufarbeiten nach a); Ausb. $2.56 \mathrm{~g} \mathrm{(92 \% )}$ 5-(Benzyloxy)-1-nitronaphthalin (30), Schmp. $100-105^{\circ} \mathrm{C}(\mathrm{EtOH})$. ${ }^{1} \mathrm{H}-\mathrm{NMR}\left(\mathrm{CDCl}_{3}\right): \delta=5.32\left(\mathrm{~s}, 2 \mathrm{H}, \mathrm{CH}_{2} \mathrm{C}_{6} \mathrm{H}_{5}\right), 7.03(\mathrm{~d}, J=8 \mathrm{~Hz}$, $1 \mathrm{H}), 7.38-7.80(\mathrm{~m}, 7 \mathrm{H}), 8.03-8.40(\mathrm{~m}, 2 \mathrm{H}), 8.60$ (dd, $J=9 \mathrm{~Hz}$, $J=1.5 \mathrm{~Hz}, 1 \mathrm{H})$.

$$
\begin{array}{lll}
\mathrm{C}_{17} \mathrm{H}_{13} \mathrm{NO}_{3} \text { (279.3) } & \text { Ber. C } 73.11 \text { H } 4.69 \text { N } 5.01 \\
& \text { Gef. C } 73.09 \text { H } 4.79 \text { N } 4.99
\end{array}
$$

Umsetzung von 1,8-Dinitronaphthalin (31): Aus $2.18 \mathrm{~g}(10.0 \mathrm{mmol})$ $31,1.00 \mathrm{~g}$ Kaliumhydroxid, $0.32 \mathrm{~g}$ TBB und $1.35 \mathrm{~g}(12.5 \mathrm{mmol})$ Benzylalkohol in $10 \mathrm{ml} \mathrm{TMH}$. Nach $5 \mathrm{~h}$ bei Raumtemp. wurde das Reaktionsgemisch auf Eis gegossen, zweimal mit jeweils $50 \mathrm{ml}$ Dichlormethan ausgeschüttelt, der Extrakt mit Magnesiumsulfat getrocknet, im Rotationsverdampfer eingeengt und der Rückstand mit Dichlormethan an Kieselgel chromatographiert; Ausb. $0.08 \mathrm{~g}$ (3\%) 1-(Benzyloxy)-4,5-dinitronaphthalin (32 b), Schmp. $168^{\circ} \mathrm{C}$ (EtOH). $-{ }^{1} \mathrm{H}-\mathrm{NMR}\left(\left[\mathrm{D}_{6}\right] \mathrm{DMSO}\right): \delta=5.53\left(\mathrm{~s}, 2 \mathrm{H}, \mathrm{CH}_{2} \mathrm{C}_{6} \mathrm{H}_{5}\right)$, $7.41-7.50(\mathrm{~m}, 4 \mathrm{H}), 7.59-7.62(\mathrm{~m}, 2 \mathrm{H}), 7.89(\mathrm{t}, J=8.45 \mathrm{~Hz}, 1 \mathrm{H})$, $8.47-8.53(\mathrm{~m}, 2 \mathrm{H}), 8.71(\mathrm{dd}, J=8.47 \mathrm{~Hz}, J=1.25 \mathrm{~Hz}, 1 \mathrm{H})$.

$$
\mathrm{C}_{17} \mathrm{H}_{12} \mathrm{~N}_{2} \mathrm{O}_{5} \text { (324.3) Ber. C } 62.96 \text { H } 3.73 \text { N } 8.64
$$

Gef. C 62.81 H 3.80 N 8.27

Die wäßrige Phase des auf Eis gegossenen Reaktionsgemischs wurde mit konz. $\mathrm{HCl}$ angesäuert und dann wie voranstehend beschrieben aufgearbeitet; Ausb. $0.07 \mathrm{~g} \mathrm{(3 \% )} \mathrm{1,8-Dinitro-2-naphthol}$ (32a), Schmp. $198-199^{\circ} \mathrm{C}$ (Zers.) $\left(\mathrm{CHCl}_{3}\right)\left(\mathrm{Lit}^{66)} 200-201^{\circ} \mathrm{C}\right),{ }^{6} \mathrm{H}$ NMR identisch mit dem in Lit. ${ }^{66)}$ und $0.52 \mathrm{~g} \mathrm{(22 \% )} \mathrm{4,5-Dinitro-1-}$ naphthol (33), Schmp. $196^{\circ} \mathrm{C}$ (Zers.) $\left(\mathrm{CHCl}_{3}\right)\left(\right.$ Lit. $^{66)} 206-208^{\circ} \mathrm{C}$ ), ${ }^{1} \mathrm{H}-\mathrm{NMR}$ identisch mit dem in Lit. ${ }^{66)}$.

Umsetzung von 1-Chlor-5-nitronaphthalin (34): $1.04 \mathrm{~g}(5.0 \mathrm{mmol})$ 34, $0.50 \mathrm{~g}$ Kaliumhydroxid, $0.16 \mathrm{~g}$ TBB und $0.65 \mathrm{~g}$ (6.0 mmol) Benzylalkohol in $7 \mathrm{ml} \mathrm{TMH}$ wurden gerührt ( $24 \mathrm{~h}$ bei Raumtemp. und $4 \mathrm{~h}$ bei $\left.50^{\circ} \mathrm{C}\right)$ und aufgearbeitet; Ausb. $1.18 \mathrm{~g} \mathrm{(88 \% )} \mathrm{5-(Benzyloxy)-}$ 1-chlornaphthalin (35), Schmp. 62-63 ${ }^{\circ} \mathrm{C}(\mathrm{MeOH}) .-{ }^{1} \mathrm{H}-\mathrm{NMR}$ $\left(\mathrm{CDCl}_{3}\right): \delta=5.25\left(\mathrm{~s}, 2 \mathrm{H}, \mathrm{CH}_{2} \mathrm{C}_{6} \mathrm{H}_{5}\right), 6.95(\mathrm{~d}, J=7.6 \mathrm{~Hz}, 1 \mathrm{H})$, 
$7.33-7.54(\mathrm{~m}, 7 \mathrm{H}), 7.59$ (dd, $J=7.4 \mathrm{~Hz}, J=1.1 \mathrm{~Hz}, 1 \mathrm{H}), 7.86$ $(\mathrm{dd}, J=9 \mathrm{~Hz}, J=0.8 \mathrm{~Hz}, 1 \mathrm{H}), 8.29(\mathrm{dt}, J=8.5 \mathrm{~Hz}, J=1 \mathrm{~Hz}$, $1 \mathrm{H})$.

\section{$\mathrm{C}_{17} \mathrm{H}_{13} \mathrm{ClO}$ (268.7) $\quad$ Ber. C $75.98 \mathrm{H} 4.88 \mathrm{Cl} 13.19$ Gef. C 76.15 H 4.98 Cl 13.29}

Umsetzung von 1-Chlor-8-nitronaphthalin (36): $2.08 \mathrm{~g}$ (10.0 mmol) 36, $1.00 \mathrm{~g}$ Kaliumhydroxid, $0.32 \mathrm{~g}$ TBB und $1.25 \mathrm{~g}$ Benzylalkohol in $10 \mathrm{ml} \mathrm{TMH}$ wurden bei $50^{\circ} \mathrm{C}$ gerührt. Nach $8 \mathrm{~h}, 24 \mathrm{~h}$ und $48 \mathrm{~h}$ wurden jeweils $0.30 \mathrm{~g}$ ( $2.8 \mathrm{mmol})$ Benzylalkohol zugegeben, danach wurde noch $8 \mathrm{~h}$ bei $80^{\circ} \mathrm{C}$ gerührt und nach b) aufgearbeitet; Ausb. nach 2maligem Chromatographieren $0.40 \mathrm{~g} \mathrm{(15 \% )} \mathrm{8-(Benzyloxy)-}$ 1-chlornaphthalin (37) farbloses Öl. $-{ }^{1} \mathrm{H}-\mathrm{NMR}\left(\mathrm{CDCl}_{3}\right)$ : $\delta=5.24$ (s, $\left.2 \mathrm{H}, \mathrm{CH}_{2} \mathrm{C}_{6} \mathrm{H}_{5}\right), 6.97$ (dd, $J=7.6 \mathrm{~Hz}, J=1.1 \mathrm{~Hz}, 1 \mathrm{H}$ ), $7.27-7.47(\mathrm{~m}, 6 \mathrm{H}), 7.51(\mathrm{dd}, J=7.5 \mathrm{~Hz}, J=1.3 \mathrm{~Hz}, 1 \mathrm{H}), 7.58$ $(\mathrm{dt}, J=6 \mathrm{~Hz}, J=1.5 \mathrm{~Hz}, 2 \mathrm{H}), 7.69(\mathrm{dd}, J=8 \mathrm{~Hz}, J=1.1 \mathrm{~Hz}$, $1 \mathrm{H})$.

$$
\begin{array}{ll}
\mathrm{C}_{17} \mathrm{H}_{13} \mathrm{ClO} \text { (268.7) } & \text { Ber. C } 75.98 \text { H } 4.88 \mathrm{Cl} 13.19 \\
& \text { Gef. C } 75.97 \text { H } 4.96 \mathrm{Cl} 13.24
\end{array}
$$

\section{CAS-Registry-Nummern}

1a: $99-65-0$ / 2b: 6831-82-9 / 2 $\mathbf{b}^{\prime}: 683-60-3$ / 2c: $865-47-4 / 2 \mathbf{c}^{\prime}$ : 865-48-5 / 2d: 22379-62-0 / 2d': 20194-18-7/2e: 100-67-4 / 3a: 555-63-3 / 3b: 88991-53-1/3c: 128924-10-7 / 3d: 24318-00-1 / 4: 554-84-7/5a: 51-28-5 / 5c: 33686-26-3 / 6a: 402-67-5/6b: $121-$ 73-3 / 6c: 585-79-5 / 6d: 645-00-1 / 6e: 18513-19-4 / 6f: 619-24-9/ 7a: 72216-35-4 / 7b: 24318-02-3/7c: 53087-13-1/7d: 10762321-2 / 7e: 128923-92-2 / 7f: 61147-43-1 / 9a: 99-35-4 / 9b: 618 86-0/9c: 18242-39-2 /9d: 618-62-2/9e: 6311-60-0/10a: $128923-$ 97-7/10b: 128923-98-8 / 10c: 128923-99-9/10d: 128924-00-5/ 10e: 128924-01-6 / 11a: 128924-02-7 / 11b: 128924-03-8 / 11c: 128924-04-9 / 12: 1528-74-1 / 13a: 2143-90-0 / 13d: 128924-05-0 / 13e: $128924-06-1 / 14 a^{\prime}: 5188-07-8 / 14 e: 3111-52-2 /$ 14f' $^{\prime}: 811$ 51-8 / 15 a: 128924-07-2 / 15e: 128924-08-3 / 15f: 128924-09-4 / 16 606-81-5 / 17: 6460-91-9 / 18: 2436-96-6 / 19: 6460-92-0 / 20: 7391 72-2 / $21: 96922-97-3$ / 22: 6311-43-9 / 23 : 53059-31-7 / 24: 107624 96-4 / 25: 958-96-3 / 26: 128923-93-3 / 27: 92-93-3 / 28: 613-37-6 29: 605-71-0 / 30: 128923-94-4 / 31: 602-38-0/32a: 80651-02-1/ 32b: 128924-11-8 / 33: 80651-03-2 / 34: 605-63-0 / 35: 128923 95-5 / 36: 602-37-9 / 37: 128923-96-6

1) 1a) M. Koch, Diplomarbeit, Univ. Stuttgart, 1985 - ${ }^{16)}$ M. Koch, Dissertation, Univ. Stuttgart, 1988.

2) W. Streicher, Dissertation, Univ. Stuttgart, 1983.

3) F. Effenberger, W. Streicher, Chem. Ber. 157; voranstehend.

4) 4a) J. R. Beck, Tetrahedron 34 (1978) 2057; Zusammenfassung, dort weitere Literaturzitate. - ${ }^{4 b)}$ G. I. Migecker, V. A. Damilento, Chem. Heterocycl. Compd. 18 (1982) 649. - 4c) N. Kornblum, L. Cheng, R. C. Korber, M. M. Kestner, B. N. Newton, H. W. Pinnick, R. G. Smith, P. A. Wade, J. Org. Chem. 41 (1976) 1560. - ${ }^{4 d)}$ F. Montanari, M. Pelosi, F. Rolla, Chem. Ind. London 12 (1982) 412 [Chem. Abstr. 97 (1982) 162484r].

5) Sał J. R. Beck, J. A. Yahner, J. Org. Chem. 43 (1978) 2048, 2052. - ${ }^{5 b)}$ P. Cogolli, L. Testaferri, M. Tingoli, M. Tiecco, J. Org. Chem. 44 (1979) 2636.

6) J. H. Gorvin, J. Chem. Soc., Chem. Commun. 1985, 238.

7) 7a) D. J. Milner, Synth. Commun. 15 (1985) 479. - ${ }^{\text {7b) }}$ G. Iwasaki, S. Saeki, M. Hamana, Chem. Lett. 1986, 31.

8) 8at J. I. G. Cadogan, D. J. Sears, D. M. Smith, J. Chem. Soc. C 1969, 1314. - ${ }^{8 b)}$ P. D. Onys'ko, Yu. G. Gololobov, G. Ya. Remennikov, V. M. Cherkasov, Khim. Geterotsikl. Soedin. 1980 , 124 [Chem. Abstr. 92 (1980) 214500].

9) M. Attina, F. Cacace, A. P. Wolf, J. Chem. Soc, Chem. Commun. 1983, 108

${ }^{10)}{ }^{10 a}$ D. W. Lamson, P. Ulrich, R. O. Hutchins, J. Org. Chem. 38 (1973) 2928 - 106) Y. Okamoto, S. T. Attarwala, J. Org. Chem. 44 (1979) 3269.

11) 11a) D. H. Rosenblatt, W. H. Dennis, R. D. Goodin, J. Am. Chem. Soc. 95 (1973) 2133. - 11b) T. J. Broxton, D. M. Muir, A. J. Parker, J. Org. Chem. 40 (1975) 2037, 3230. - iic) M. Kobayashi, T. Sakai, H. Minato, Chem. Lett. 1975, 1253. - ${ }^{1 \mathrm{td})}$ J. Hayami, M. Asahi, R. Tamura, N. Ono, Bull. Inst. Chem. Res., Kyoto Univ. 58 (1980) 222 [Chem. Abstr. 94 (1981) 46372s].
12) 12a) E. Buncel, M. Hamaguchi, A. R. Norris, J. Chem. Soc., Perkin Trans. 1, 1980, 2205. - ${ }^{126)}$ A. J. Elliot, M. S. Gibson, J. Org. Chem. 45 (1980) 3677. - ${ }^{12 c)}$ K. Rasheed, J. D. Warkentin, J. Org. Chem. 45 (1980) 4041.

13) 13a) E. Radlmann, W. Schmidt, G. E. Nischk, Macromol. Chem. 130 (1969) 45. - ${ }^{13 b)}$ H. M. Relles, C. M. Orlando, D. R. Heath, R. W. Schluenz, J. S. Manello, S. Hoff, J. Polym. Sci., Polym. Chem. Ed. 15 (1977) 2441. - ${ }^{13 c)}$ T. Takekoshi, J. G. Wirth, D. R. Heath, J. E. Kochanowski, J. S. Manello, M. J. Webber, J. Polym. Sci., Polym. Chem. Ed. 18 (1980) 3069.

14) F. Effenberger, Angew. Chem. 92 (1980) 147; Angew. Chem. Int. Ed. Engl. 19 (1980) 151.

15) H. Limpricht, Liebigs Ann. Chem. 117 (1875) 60.

16) 16a) I. G. Farbenindustrie, D.R.P. 513798 (1928); Friedländer 17 767. - ${ }^{166)}$ Ube Industries, Ltd. (K. Yamada, M. Nakahigashi, S. Nagai, H. Yamane, A. Takaoka, K. Harada, H. Yoshida, T. Konishi, Erf.), Jpn. Kokai Tokkyo Koho JP. 72 38,834 (6. Dez. 1972) [Chem. Abstr. 78 (1973) P58028s].

17) 17a) J. Cervello, M. Figueredo, J. Marquet, M. Moreno-Mañas, J. Bertrám, J. H. Lluch, Tetrahedron Lett. 1984, 4147. - ${ }^{17 b)} \mathrm{P}$. Brasem, J. C. Lammers, J. Cornelisse, J. Ingstenburg, E. Haringa, Tetrahedron Lell. 1972, 685.

18) 18a) Mitsui Toatsu Chemicals, Inc. (T. Toyota, F. Matsuda, Erf.), Jpn. Kokai Tokkyo Koho JP. 79 39,030 (24. März 1979) [Chem. Abstr. 91 (1979) P 20104 u]. - ${ }^{18 b)}$ Mitsui Toatso Chemicals, Inc., Jpn. Kokai Tokkyo Koho JP. 58,180,461 [83,180,461] (21. Okt. 1983) [Chem. Abstr. 100 (1984) P 102924e] - ${ }^{18 c)}$ Mitsui Toatsu Chemicals, Inc., Jpn. Kokai Tokkyo Koho JP. 59,25,353 [84,25,353] (2. Aug. 1982) [Chem. Abstr. 101 (1984) P 23116k] - ${ }^{18 d)}$ Mitsui Toatsu Chemicals, Inc., Jpn. Kokai Tokkyo Koho JP. 59,44,343 [84,44,343] (12. März 1984) [Chem. Abstr. 101 (1984) P $110518 \mathrm{~m}]$.

19) Mitsui Toatsu Chemicals, Inc., Jpn. Kokai Tokkyo Koho JP. 59,157,059 [84,157,059] (6. Sept. 1984) [Chem. Abstr. 102 (1985) $\mathrm{P} 45613 \mathrm{t}]$

${ }^{20)}{ }^{20 a)}$ H. S. Fry, J. L. Cameron, J. Am. Chem. Soc. 49 (1927) 864. 20b) Y. Ogata, J. Mibae, J. Org. Chem. 27 (1962) 2048.

21) W. J. van Zoest, H. J. den Hertog, Recl. Trav. Chim. Pays-Bas 93 (1974) 166.

22) 22a) A. Wohl, Ber. Dtsch. Chem. Ges. 32 (1899) 3486. - ${ }^{22 b)}$ E. V. Malykhin, G. A. Kolesnichenko, V. D. Shteingarts, Zh. Org. Khim. 22 (1986) 806; J. Org. Chem. USSR (Engl. Transl.) 22 (1986) 720

23) R, D. Guthrie, D. E. Nutter, J. Am. Chem. Soc. 104 (1982) 7478 24) 24a) C. F. Bernasconi, Chimia 34 (1980) 1. - ${ }^{24 b)}$ J. F. Bunnett, Acc. Chem. Res. $11(1978)$ 413. - ${ }^{24 c)}$ R. D. Guthrie, C. Hartmann, R. Neill, D. E. Nutter, J. Org. Chem. 52 (1987) 736.

25) 25a) E. N. Omelechko. V. A. Ryabinin, S. M. Shein, Zh. Org. Khim. 18 (1982) 1123; J. Org. Chem. USSR (Engl. Transl.) 18 (1982) 972. - ${ }^{25 b)}$ G. A. Russel, A. G. Bemis, Inorg. Chem. 6 (1967) 403. - ${ }^{25 c)}$ A. Bassani, M. Prato, P. Rampazzo, U. Quintily, G. Seorrano, J. Org. Chem. 45 (1980) 2263. - ${ }^{25 d)}$ A. Frimer, I. Rosenthal, Tetrahedron Lett. 1976, 2809.

26) A. F. Holleman, J. W. Beekman, Recl. Trav. Chim. Pays-Bas 23 (1904) 225

27) 27a) E. B. Pedersen, J. Perregard, S. O. Lawesson, Tetrahedron 29 (1973) 4211. - 276) J. T. Gupton, J. P. Idoux, B. Baker, C. Colon, A. D. Crews, C. D. Jurss. R. C. Rampi, J. Org. Chem. 48 (1983) 2933. - ${ }^{27 c)}$ Hitachi Ltd., Hitachi Chemical Co., Jpn. Kokai Tokkyo Koho, JP. 58,180,530 [83,180,530] (22. Okt. 1983) [Chem. Abstr. 100 (1984) P 139808 w].

${ }^{28)}{ }^{28 a}$ A. P. Kozikowskii, M. N. Greco, J. P. Springer, J. Am. Chem. Soc. 104 (1982) 7622.

29) V. D. Parker, Acta Chem. Scand., Ser. B, 35 (1981) 655.

30) 30a) J. P. Idoux, M. L. Madenwald, B. S. Garcia, D.-L. Chu, J. T. Gupton, J. Org. Chem. 50 (1985) 1876. - ${ }^{30 b)}$ V. A. Ustinov, V. V. Plakhtinskii, G. S. Mironov, N. S. Ryabukhina, Osnovn. Org. Sint. Neftekhim. 1979, 54 [Chem. Abstr, 93 (1980) 45509 j].

31) 31a) V. Gold, C. H. Rochester, J. Chem. Soc., Perkin Trans. 2, $1964,1692 .{ }^{31 b)}$ S. S. Gitis, I. G. L'vovich, $Z h$. Obshch. Khim. 34 (1964) 2250; J. Gen. Chem. USSR (Engl. Transl.) 34 (1964) 2262.

32) M.A.C. De Kock, Recl. Trav. Chim. Pays-Bas 20 (1901) 112. 32b) V. N. Kugazev, V. N. Drozd, A. A. Klinov, Zh. Org. Khim. 12 (1976) 2387; J. Org. Chem. USSR (Engl. Transl.) 12 (1976) 2319.

${ }^{33)}$ V. N. Boiko, G. M. Shchupak, Zh. Org. Khim. 13 (1977) 1042; J. Org. Chem. USSR (Engl. Transl.) 13 (1977) 958.

${ }^{34)}$ F. Reverdin Org. Synth., Coll. Vol. I (1948) 219. 
${ }^{35)}$ C. F. Bernasconi, J. Am. Chem. Soc. 92 (1970) 4682.

36) P. T. Izzo, J. Org. Chem. 24 (1959) 2026.

37) 37a! J. R. Beadle, S. H. Korzeniowski, D. E. Rosenberg, B. J. Garcia-Slanga, G. W. Gokel, J. Org. Chem. 49 (1984) 1594. 37b) M. Koch, J. R. Beadle, S. H. Korzeniowski, D. E. Rosenberg, B. J. Garcia-Slanga, G. W. Gokel, Bull. Acad. Sci. USSR, Div. Chem. Sci. (Engl. Transl.) 30 (1981) 1993.

${ }^{381}$ N. A. Bumagin, I. O. Kalinowskii, I. P. Beletskaya, Izv. Akad. Nauk SSR, Ser. Khim. 1981, 2413 [Chem. Abstr. 96 (1982) $68474 \mathrm{v}]$

39) ${ }^{39 a)}$ H. H. Hodgson, H. E. Marschen, J. Chem. Soc. 1940, 208. -

${ }^{39 b)}$ H. C. Gull, E. E.. Turner, J. Chem. Soc. 1929, 494. - ${ }^{39 c)}$ F.H. Case, A. Idelson, J. Org. Chem. 27 (1962) 4651. - ${ }^{39 d)}$ F. Ullmann, J. Bielecki, Ber. Dtsch. Chem. Ges. 34 (1901) 2174

${ }_{40)}^{40 a)}$ F. Bell, J. Kenyon, J. Chem. Soc. 1926, 3044. - ${ }^{40 \mathrm{~b})}$ J. P. Idoux, V. S. Cantwell, J. Hinton, S. O. Nelson, P. Hollier, P. Zarrillo, J. Org. Chem. 39 (1974) 3946.

41) 41a) E. Berliner, B. Newman, T. M. Riaboff, J. Am. Chem. Soc. 77 (1955) 478 - - ${ }^{41 b i}$ C. Dell'Erba, G. Guanti, G. Garbarino, Tetrahedron 27 (1971) 1807. - ${ }^{41 c)}$ D. J. Byron, G. W. Gray, R. C. Wilson, J. Chem. Soc. C, 1966, 831.

${ }^{42)}$ V. A. Koptyug, T. N. Geratimova, A. A. Tabatskaya, Izv. Sib. Otd. Akad. Nauk SSSR, Ser. Khim. Nauk, 1966, 62 [Chem. Abstr. 67 (1967) $32228 \mathrm{e}]$.

43) 43a) W. L. Hinze, L.-J. Liu, J. H. Fendler, J. Chem. Soc., Perkin Trans. 2, 1975, 1751. - ${ }^{436)}$ E. V. Malykhin, A. A. Shtark, V. D. Shteingarts, Zh. Org. Khim. 18 (1982) 1898; J. Org. Chem. USSR (Engl. Transl.) 18 (1982) 1661. - ${ }^{43 c)}$ Yu. M. Atroshchenko, S S. Gitis, A. Ya. Kaminskii, Zh. Org. Khim. 25 (1989) 214; J. Org. Chem. USSR (Engl. Transl.) 25 (1989) 195.

${ }^{44)}{ }^{44 a)} \mathrm{H}$. Meerwein in Methoden der Organischen Chemie (HoubenWeyl-Müller), 4.Aufl., Bd. VI/3, S. 75, Thieme, Stuttgart 1965. 446) H. R. Todd, R. L. Shriner, J. Am. Chem. Soc. 56 (1934) 1382

${ }^{45)} \mathrm{R}$. Kaiser, Chromatographie in der Gasphase, Bd. 4, S. 206, Bibliographisches Institut, Mannheim, 1965.

${ }^{46)}$ B. Glatz, Dissertation, Univ. Stuttgart 1976.
47) R. C. Weast, M. J. Astle, CRC Handbook of Chemistry and Physics, 63. Aufl., C-136, CRC Press Inc., Boca Raton, Florida $1982-1983$.

48) B. Jones, J. Chem. Soc. 1943, 430.

49) H. H. Hodgson, H. Clay, J. Chem. Soc. 1932, 869

(5) F. Pietra, D. Vitali, F. Del Cima, G. Cardinali, J. Chem. Soc. B $1970,1659$.

51) Lit. ${ }^{43)}, \mathrm{C}-437$

52) Lit. ${ }^{47)}, \mathrm{C}-435$.

53) G. G. Yacobson, N. N. Vorozhtsov, Zh. Vses. Khim. Ova. $6(1961)$ 360 [Chem. Abstr. 55 (1961) 27172e].

${ }^{54)}$ F. Arnall, J. Chem. Soc. 1924, 811.

${ }^{55)}$ S. I. Pombrik, A. S. Peregudov, D. N. Kravtsov, Bull. Acad. Sci. USSR, Div. Chem. Sci. (Engl. Transl.) 34 (1985) 1189; Izv. Akad. Nauk SSSR, Ser. Khim. 1985, 1300.

56) Y. H. Wu, W. A. Gould, W. G. Lobek, Jr., H. R. Roth, R. F Feldkamp, J. Med. Pharm. Chem. 5 (1962) 752 [Chem. Abstr. 57 (1962) $12412 d]$

57) Troponwerke Dinklage und Co. (C. Schöpf, Erf.), Ger. 925,475 (21. März 1955) [Chem. Abstr. 52 (1958) 3861 a].

${ }^{58)}$ N. B. Dean, W. B. Whalley, J. Chem. Soc. 1954, 4638.

${ }^{59)}$ B. Jones, J. P. Sleight, J. Chem. Soc. 1954, 1775

${ }^{60)}$ R. A. Abramovitch, O. A. Koleoso, J. Chem. Soc. B, 1969, 779.

${ }^{61)}$ M. Nilsson, Acta Chem. Scand. 20, 423.

${ }^{62)}$ W. J. Dunstan, G. K. Hughes, J. Proc. R. Soc. N. S. W. 80 (1947) 77 [Chem. Abstr. 42 (1948) 4161 a].

63) L. G. Makarova, M. K. Matveeva, E. A. Gribchenko, Bull. Acad. Sci. USSR, Div. Chem. Sci. (Engl. Transl.) 7 (1958) 1399 [Chem. Abstr. 53 (1959) 8057c7.

${ }^{64)}$ T. Migita, R. Ito, K. Tori, O. Shimamura, Yuki Gosei Kagaku Kyokaishi 19 (1961) 605 [Chem. Abstr. 55 (1961) 24667a].

${ }^{65)}$ S. H. Korzeniowski, L. Blum, G. W. Gokel, Tetrahedron Lett. 1977, 1871

66) E. V. Malykhin, V. D. Shteingarts, Zh. Org. Khim. 17 (1981) 2402; J. Org. Chem. USSR (Engl. Transl.) 17 (1981) 2146.

$[169 / 90]$ 